\title{
Multiphase phase field theory for temperature-induced phase transformations: formulation and application to interfacial phases
}

\author{
Valery I. Levitas ${ }^{1}$ and Arunabha M. Roy ${ }^{2}$ \\ ${ }^{1}$ Iowa State University, Departments of Aerospace Engineering, Mechanical Engineering, and \\ Material Science and Engineering, Ames, Iowa 50011, U.S.A. \\ ${ }^{2}$ Iowa State University, Department of Aerospace Engineering, Ames, Iowa 50011, U.S.A.
}

The main conditions for the thermodynamic potential for multiphase Ginzburg-Landau theory are formulated for temperature-induced phase transformations (PTs). Theory, which satisfies all these conditions for $n$-phase material, is developed. The key point is a new penalizing term in the local energy that allows controlling absence or presence and the extent of the presence of the third phase within the interface between two other phases. A finiteelement method is applied for studying $\mathrm{PT}$ between $\beta$ and $\delta$ phases of HMX energetic crystal via intermediate melting more than $100^{\circ} \mathrm{C}$ below melting temperature. Depending on material parameters (ratio of the width and energy of the solid-solid $(S S)$ to solid-melt interface and the magnitude of the penalizing term), there are either two (meta)stable stationary interfacial nanostructures, corresponding to slightly and strongly disordered interfaces (in the limits, pure $S S$ interface or complete melt within $S S$ interface), or these nanostructures coincide. A parametric study of these nanostructures is presented. The developed requirements and approach are applicable to various PTs between multiple solid and liquid phases and can be elaborated for PTs induced by mechanical and electromagnetic fields, diffusive PTs, and the evolution of multi-grain and multi-twin microstructures.

\section{Introduction}

While in this paper we focus on the temperature-induced multiphase PTs, we will mention some works which include stresses as well, because these theories reduce to the temperature-induced PTs at zero stresses. The main focus is on the description of the first-order PTs for the case when a PT completes and there are no structural changes after completing PT, like for melting, martensitic PTs, and some reconstructive PTs. The main problem is to develop a consistent phase field approach (PFA) for PTs between an arbitrary number of phases. There are two very different approaches with different goals developed by two different communities. The first 
one is favored within the community working on the description of PTs between the austenite (A) and any of the $n$ martensitic variants $\mathrm{M}_{i}$ and between martensitic variants $\mathrm{M}_{j} \leftrightarrow \mathrm{M}_{i}$ (which represents twinning in most cases) [1-8]. It utilizes $n$ independent order parameters $\eta_{i}$, each of which describes $\mathrm{A} \leftrightarrow \mathrm{M}_{i}$ PTs between $n+1$ phases. In most papers, researchers work within this approach at the actual spatial scales, rather than within coarse-grained theories for the microscale. Thus, typical actual interface width is on the order of nanometers and detail of distribution of all parameters within the interface are of interest. That is why all simulations are limited to submicron samples.

The second multiphase approach is developed within the community working on multiphase solidification (e.g., in eutectic and peritectic systems) and grain growth [9-18]. It operates with $n+1$ order parameters $\eta_{i}$ satisfying constraint $\sum \eta_{i}=1$, similar to phase concentrations. In most of these theories interface width artificially increased by several orders of magnitude (see, e.g., $[11,16,17]$ or microscale theories $[19,20])$, and detail of variation of material parameters and fields across an interface are unrealistic but this is not important for the chosen objectives. This is done in order to be able to treat much larger samples comparable to those relevant for studying solidification of actual materials.

Each of these approaches satisfies some important requirements formulated to achieve some specific goals and have their advantages and drawbacks. They will be analyzed in Section 4 and it will be shown that none of them meets all the desired requirements. Two of the requirements, which were imposed in the second approach and ignored in the first approach, are that each of the two-phase PTs should be described by a single order parameter and that an interface between any of the two phases should not contain the third phase [16-18,21]. These conditions are, in particular, required in order to have the possibility to obtain an analytical solution for a propagating interface, which can be used to calibrate parameters of the thermodynamic potential in terms of interface energy, width, and mobility that are assumed to be known. In the coarse-grained approach computational interface width is usually used, which may be larger than the physical width by several orders of magnitude, but keeps the same (i.e., independent of the interface width) energy and mobility. If the order parameter corresponding to the third phase appears within an interface between two other phases, then (as it follows from the thininterface consideration $[16,17,22,23])$ a solution depends on the interface width, which due to 
unphysical width leads to incorrect results. Thus, PT between each of the two phases should occur along the straight line (or any line, which is independent of temperature, e.g., circle [8, 24$27])$ in the order parameter space. Since a single constraint $\sum \eta_{i}=1$ does not lead to such a transformation path, additional efforts are made to satisfy these two conditions [16-18]. These efforts, however, do not completely solve the problem either. Note that the requirement that PT criteria should follow from the thermodynamic instability conditions accepted in $[1,2,8]$ for the first approach was never used for the second approach [9, 11, 16-18]. Hyperspherical order parameters and a nonlinear constraint were suggested in $[8,28]$ for multivariant martensitic PTs.

In the paper, we explicitly formulate all requirements, which we want to satisfy, first for two-phase PFA, then for an arbitrary number of phases. Then we develop a theory, which satisfies all these requirements. Instead of imposing constraints on the order parameter, we introduce simple terms penalizing deviation of the paths in the order parameter space from the straight lines connecting each of the two phases. By controlling these terms, we can either fully avoid a third phase within an interface between two other phases or allow it in order to describe the actual physical situation [24-27, 29-31]. Comparison with previous requirements is performed. A number of model problems for a solid-solid PT via intermediate melting (IM) in HMX energetic material are solved and analyzed. Note that a similar approach, but without proper justification and with emphases on stress-induced PTs and twinning, when the third phase is excluded from the interface between two other phases, was presented in [32]. Detailed comparison of existing model with present model was presented in Appendix A.

\section{Two-phase model}

\subsection{Ginzburg-Landau equation}

The free energy $\psi$, dissipation rate $D$ (both per unit mass), and Ginzburg-Landau equation for a single order parameter $\eta$ have the form

$$
\begin{aligned}
& \psi=\psi^{\theta}(\theta, \eta)+0.5 \beta|\nabla \eta|^{2} ; \quad D=X \dot{\eta} \geq 0 \\
& \dot{\eta}=L X=-L \frac{\delta \psi}{\delta \eta}=L\left(-\frac{\partial \psi^{\theta}}{\partial \eta}+\beta \boldsymbol{\nabla}^{2} \eta\right)
\end{aligned}
$$

where $\psi^{\theta}$ is the local thermal (chemical) energy, $\beta>0$ and $L>0$ are the gradient energy 
and kinetic coefficients, $X$ is the thermodynamic driving force conjugate to $\dot{\eta}$, and $\frac{\delta}{\delta}$ is the variational derivative. Our goal is to formulate requirements to $\psi^{\theta}(\theta, \eta)$ and some interpolation functions and find the simplest function that satisfies these requirements. Since all requirements are for homogeneous states, the gradient-related term in $X$ can be omitted.

\subsection{Conditions for free energy for bulk phases}

1. We would like to enforce that $\eta=0$ corresponds to the phase $\mathrm{P}_{0}$ and $\eta=1$ corresponds to the phase $\mathrm{P}_{1}$. It is convenient to express any material property $M$ (energy, entropy, specific heat, and when mechanics is included, also elastic moduli and thermal expansion) in the form

$$
M(\eta, \theta)=M_{0}(\theta)+\left(M_{1}(\theta)-M_{0}(\theta)\right) \varphi_{m}(\eta)
$$

where $M_{0}$ and $M_{1}$ are values of the property $M$ in phases $\mathrm{P}_{0}$ and $\mathrm{P}_{1}$, respectively, and $\varphi_{m}(\eta)$ is the corresponding interpolation function, which satisfies evident conditions

$$
\varphi_{m}(0)=0, \quad \varphi_{m}(1)=1
$$

In application to free energy, we obtain

$$
\psi^{\theta}(\theta, 0)=\psi_{0}^{\theta}(\theta), \quad \psi^{\theta}(\theta, 1)=\psi_{1}^{\theta}(\theta)
$$

where $\psi_{0}^{\theta}(\theta)$ and $\psi_{1}^{\theta}(\theta)$ are the free energies of the bulk phases $\mathbf{P}_{0}$ and $\mathbf{P}_{1}$. However, it is not sufficient to verbally impose that $\eta=0$ corresponds to the phase $\mathrm{P}_{0}$ and $\eta=1$ corresponds to the phase $P_{1}$. This should directly follow from the thermodynamic equilibrium conditions, because bulk phases should be thermodynamic equilibrium solutions of the Ginzburg-Landau Eq.(2).

2. Values $\eta=0$ and $\eta=1$ should satisfy the thermodynamic equilibrium conditions

$$
X=-\frac{\partial \psi^{\theta}(\theta, 0)}{\partial \eta}=-\frac{\partial \psi^{\theta}(\theta, 1)}{\partial \eta}=0
$$

for any temperature $\theta$. Otherwise, thermodynamic equilibrium values of the order parameters obtained from condition $X=0$ will depend on temperature. Substituting them in Eq.(3) will introduce artificial temperature dependence of the property $M$ and will not allow us to obtain known properties $M_{0}$ and $M_{1}$ for bulk phases $\mathrm{P}_{0}$ and $\mathrm{P}_{1}$. It also follows from Eq.(6) that for any material property which participates in $\psi^{\theta}$, one has

$$
\frac{d \varphi_{m}(0)}{d \eta}=\frac{d \varphi_{m}(0)}{d \eta}=0 .
$$


3. The free energy should not possess unphysical minima for any temperature. Any minimum in the free energy that does not correspond to the desired minima for phases $P_{0}$ and $P_{1}$ represents a spurious (unphysical) phase. It cannot be interpreted as a "discovery" of a new phase, because it is just consequence of chosen polynomial approximation rather than any physical knowledge. In particular, one can "discover" as many new phases as he/she wishes, if some periodic function of the order parameters is added to the potential.

The smallest degree potential that satisfies all these properties is the fourth degree. Thus, starting with the full fourth degree polynomial $\varphi=h+g \eta+a \eta^{2}+b \eta^{3}+c \eta^{4}$ and applying conditions 1-3, one obtains:

$$
\varphi(a, \eta)=a \eta^{2}+(4-2 a) \eta^{3}+(a-3) \eta^{4}=a \eta^{2}(1-\eta)^{2}+\eta^{3}(4-3 \eta),
$$

where $a$ is a parameter. If properties vary monotonously between phase, i.e., the function $\varphi(a, \eta)$ does not have an extremum on the interval $0 \leq \eta \leq 1$, then one has to impose $0 \leq a \leq 6$. Similar, starting with $\psi^{\theta}=H+G \eta+A \eta^{2}+B \eta^{3}+C \eta^{4}$, and applying conditions 1-3, we derive

$$
\psi^{\theta}(\theta, \eta)=\psi_{0}^{\theta}(\theta)+\Delta \psi^{\theta}(\theta) \eta^{3}(4-3 \eta)+A \eta^{2}(1-\eta)^{2}, \quad \Delta \psi^{\theta}=\psi_{1}^{\theta}(\theta)-\psi_{0}^{\theta}(\theta) .
$$

Here $A$ is the material parameter, which depends or may depend on temperature (the same is true for $a), \Delta \psi^{\theta}$ is the negative thermal driving force for the $\mathrm{P}_{0} \rightarrow \mathrm{P}_{1}$ phase transformation. The first two terms in $\psi^{\theta}$ represent smooth interpolation between $\psi_{0}^{\theta}$ and $\psi_{1}^{\theta}$, and the last one is a double-well barrier. Eq.(9) can give the wrong impression that the function $\eta^{3}(4-3 \eta)$ is the only interpolation function for $\Delta \psi^{\theta}$. However, $A$ may include $\Delta \psi^{\theta}$ in some way as well. Eq.(9) was obtained by excluding parameters $B$ and $C$ while imposing our constrains. However, if we exclude $A$ and $B$ or $A$ and $C$, we obtain two different expressions:

$$
\begin{aligned}
& \psi^{\theta}(\theta, \eta)=\psi_{0}^{\theta}(\theta)+\Delta \psi^{\theta}(\theta) \eta^{2}(3-2 \eta)+C \eta^{2}(1-\eta)^{2} \\
& \psi^{\theta}(\theta, \eta)=\psi_{0}^{\theta}(\theta)+\Delta \psi^{\theta}(\theta) \eta^{2}\left(2-\eta^{2}\right)-0.5 B \eta^{2}(1-\eta)^{2}
\end{aligned}
$$

satisfying the same conditions. To avoid this multiplicity of presentations, we define $\psi^{\theta}$ as the sum of the double-barrier function (which is the same in all presentations) and the most general monotonous interpolation between $\psi_{0}^{\theta}$ and $\psi_{1}^{\theta}$ satisfying conditions 1-3:

$$
\psi^{\theta}(\theta, \eta)=\psi_{0}^{\theta}(\theta)+\Delta \psi^{\theta}(\theta) \varphi(a, \eta)+A \eta^{2}(1-\eta)^{2} .
$$


Now we can exclude dependence of $A$ on $\Delta \psi^{\theta}$ without loss of generality. For a different $a$ we can obtain Eqs.(9)-(10).

4. Conditions for thermodynamic instability of equilibrium phases $P_{0}$ and $P_{1}$ should give specific instability temperatures, which are temperatures for barrierless PT or spinodal temperatures. Critical temperature should be below phase equilibrium temperature $\theta_{e}$ for hightemperature phase $\mathrm{P}_{0}$ and above $\theta_{e}$ for low temperature phase $\mathrm{P}_{1}$. As we will see, this condition imposes some restrictions for the free energy (11), but it cannot be satisfied for some popular fifth-degree potentials used in $[16,17,33]$. Thermodynamic instability conditions are

$$
\begin{gathered}
\mathrm{P}_{0} \rightarrow \mathrm{P}_{1}: \quad \partial X(\theta, 0) / \partial \eta=-\partial^{2} \psi^{\theta}(\theta, 0) / \partial \eta^{2}=-2\left(A+a \Delta \psi^{\theta}\right) \geq 0 \rightarrow-\Delta \psi^{\theta} \geq A(\theta) / a ;(12) \\
\mathrm{P}_{1} \rightarrow \mathrm{P}_{0}: \quad \partial X(\theta, 1) / \partial \eta=-\partial^{2} \psi^{\theta}(\theta, 1) / \partial \eta^{2}=-2\left(A+(a-6) \Delta \psi^{\theta}\right) \geq 0 \rightarrow-\Delta \psi^{\theta} \leq A(\theta) /(a-6),
\end{gathered}
$$

where we took into account that $a<6$. Thus, barrierless direct $\mathrm{PT} \mathrm{P}_{0} \rightarrow \mathrm{P}_{1}$ occurs when the driving force $-\Delta \psi^{\theta}$ exceeds some positive threshold and barrierless reverse $\mathrm{PT} \mathrm{P}_{1} \rightarrow \mathrm{P}_{0}$ occurs when the driving force $-\Delta \psi^{\theta}$ is smaller than some negative threshold; there is a hysteresis, which is logical and agrees with condition 4 . Let us assume that $A$ and $\Delta \psi^{\theta}$ are linear functions of temperature: $A(\theta)=A_{*} \theta-B_{*}$ and $\Delta \psi^{\theta}=-\Delta s\left(\theta-\theta_{e}\right)$, where $A_{*}$ and $B_{*}<0$ are parameters and $\Delta s=s_{1}-s_{0}$ is the jump in entropy between phases $\mathrm{P}_{1}$ and $\mathrm{P}_{0}$. We also assume that $\mathrm{P}_{0}$ is the high-temperature phase and, consequently, $\Delta s<0$. Then instability conditions (12)-(13) reduce to

$$
\begin{aligned}
& \mathrm{P}_{0} \rightarrow \mathrm{P}_{1}: \quad \theta<\theta_{c}^{0} ; \quad \theta_{c}^{0}:=\left(a \Delta s \theta_{e}-B_{*}\right) /\left(a \Delta s-A_{*}\right) ; \quad a \Delta s<A_{*} \\
& \mathrm{P}_{1} \rightarrow \mathrm{P}_{0}: \quad \theta>\theta_{c}^{1} ; \quad \theta_{c}^{1}:=\left((6-a) \Delta s \theta_{e}+B_{*}\right) /\left((6-a) \Delta s+A_{*}\right) ; \quad(a-6) \Delta s<A_{*}
\end{aligned}
$$

where $\theta_{c}^{0}$ and $\theta_{c}^{1}$ are the critical temperatures for the loss of the thermodynamic stability of phases $P_{0}$ and $P_{1}$, and $:=$ is equal by definition. The required conditions $\theta_{c}^{0}<\theta_{e}$ and $\theta_{c}^{1}>\theta_{e}$ both lead to the same restriction: $B<A_{*} \theta_{e}$. Since critical temperatures are often not wellknown, one of the plausible assumptions, $\theta_{e}=0.5\left(\theta_{c}^{0}+\theta_{c}^{1}\right)$, is used [1]. It results in two possible relationships. The first one, $A_{*}=B_{*} / \theta_{e}$, leads to $\theta_{e}=\theta_{c}^{0}=\theta_{c}^{1}$ and is physically unacceptable. The second one is $A_{*}=(a-3) \Delta s$ and results in

$$
\theta_{c}^{0}:=\left(a \Delta s \theta_{e}-B_{*}\right) /(3 \Delta s) ; \quad \theta_{c}^{1}:=\left((6-a) \Delta s \theta_{e}+B_{*}\right) /(3 \Delta s)
$$


5. Interpolating functions $\varphi(a, \eta)$ should satisfy the following antisymmetry condition:

$$
\varphi(a, 1-\eta)=1-\varphi(a, \eta)
$$

This condition is not required for a single order parameter but will be required for consistent description for a multiphase system and multiple order parameters. This condition is satisfied for $a=3$ only. Thus, the interpolating function reduces to

$$
\phi(\eta)=\varphi(3, \eta)=\eta^{2}(3-2 \eta)
$$

and instability conditions to

$$
\mathrm{P}_{0} \rightarrow \mathrm{P}_{1}: \quad-\Delta \psi^{\theta} \geq A(\theta) / 3 ; \quad \mathrm{P}_{1} \rightarrow \mathrm{P}_{0}: \quad-\Delta \psi^{\theta} \leq-A(\theta) / 3
$$

The critical temperatures are

$$
\theta_{c}^{0}:=\left(3 \Delta s \theta_{e}-B_{*}\right) /\left(3 \Delta s-A_{*}\right) ; \quad \theta_{c}^{1}:=\left(3 \Delta s \theta_{e}+B_{*}\right) /\left(3 \Delta s+A_{*}\right),
$$

and for the case when $\theta_{e}=0.5\left(\theta_{c}^{0}+\theta_{c}^{1}\right)$ one has $A_{*}=0, A$ is temperature independent, and

$$
\theta_{c}^{0}:=\theta_{e}-B_{*} /(3 \Delta s) ; \quad \theta_{c}^{1}:=\theta_{e}+B_{*} /(3 \Delta s)
$$

Condition 5 means complete equivalence of phases $P_{1}$ and $P_{0}$ in the following sense. If we consider the order parameter $\bar{\eta}=1-\eta$, which is zero for $\mathrm{P}_{1}$ and 1 for $\mathrm{P}_{0}$, then the function

$$
\phi(\bar{\eta})=\phi(1-\eta)=1-\phi(\eta)=1-\phi(1-\bar{\eta})
$$

in terms of $\bar{\eta}$ meets the same condition as in Eq.(17) in term of $\eta$. The plot of functions $\phi(\eta)$ and $\phi(\bar{\eta})$ is symmetric with respect to the vertical mirror at $\eta=\bar{\eta}=0.5$. Substituting $\eta=1-\bar{\eta}$ in Eq.(4), we obtain

$M(\eta, \theta)=M_{0}+\left(M_{1}-M_{0}\right) \phi(1-\bar{\eta})=M_{0}+\left(M_{1}-M_{0}\right)(1-\phi(\bar{\eta}))=M_{1}+\left(M_{0}-M_{1}\right) \phi(\bar{\eta})$.

Thus, all material properties and, consequently, the entire theory are invariant with respect to exchange $\left(\mathrm{P}_{0}, \eta\right) \leftrightarrow\left(\mathrm{P}_{1}, \bar{\eta}\right)$. Eq.(11) simplifies to

$$
\psi^{\theta}(\theta, \eta)=\psi_{0}^{\theta}(\theta)+\Delta \psi^{\theta}(\theta) \phi(\eta)+A \eta^{2}(1-\eta)^{2} .
$$

Condition 5 is definitely not a fundamental property and may not be true for various phase transformations. It is restrictive but this is a price that one must pay to be able to develop a 
multiphase PFA within given framework.

\section{Model with $n$ order parameters}

\subsection{Ginzburg-Landau equations}

We consider $n+1$ phases $\mathrm{P}_{0}$ and $\mathrm{P}_{i}(i=1,2, \ldots, n)$ described by $n$ order parameters $\eta_{i}$. Each $\mathrm{PT} \mathrm{P}_{0} \leftrightarrow \mathrm{P}_{i}$ is described by a single order parameter $\eta_{i}$. We designate the set of the arbitrary order parameters as $\tilde{\eta}=\left(\eta_{1}, \ldots, \eta_{i}, \ldots, \eta_{n}\right)$ with $\bar{\eta}_{i}=\left(0, \ldots, \eta_{i}, \ldots, 0\right)$ for one nonzero parameter only. The reference phase $P_{0}$ corresponds to $\hat{\eta}_{0}:=(0, \ldots, 0)$ and phase $P_{i}$ is designated as $\hat{\eta}_{i}=\left(0, \ldots, \eta_{i}=1, \ldots, 0\right)$. The generalization of Eqs.(1) and (2) for the free energy $\psi$, dissipation rate $D$, and Ginzburg-Landau equation is

$$
\begin{aligned}
& \psi=\psi^{\theta}\left(\theta, \eta_{i}\right)+\sum 0.5 \beta_{i j} \nabla \eta_{i} \cdot \nabla \eta_{j} ; \quad \beta_{i j}=\beta_{j i} ; \quad D=\sum X_{i} \dot{\eta}_{i} \geq 0 \\
& \dot{\eta}_{i}=L_{i j} X_{j}=L_{i j}\left(-\frac{\partial \psi}{\partial \eta_{j}}+\sum \beta_{j k} \nabla^{2} \eta_{k}\right) ; \quad L_{i j}=L_{j i},
\end{aligned}
$$

where $\beta_{i j}$ and $L_{i j}$ are positively defined gradient energy and kinetic coefficients, $X_{i}$ is the thermodynamic driving force conjugated to $\dot{\eta}_{i}$.

\subsection{Conditions for thermodynamic potential}

Here, we slightly generalize conditions 1-4 for $n$ order parameters and will mark them with $n$. There is no correspondence between numbers with and without $n$, it is new numeration, which includes new conditions, which do not have a counterpart for two-phase models.

1n. Any material property $M$ can be expressed in the form

$$
M\left(\eta_{i}, \theta\right)=M_{0}(\theta)+\sum\left(M_{i}(\theta)-M_{0}(\theta)\right) \phi\left(\eta_{i}\right) ; \quad \phi\left(\eta_{i}\right)=\eta_{i}^{2}\left(3-2 \eta_{i}\right)
$$

We used the simplest linear combination without interaction effects and with the interpolation function which satisfies all the requirements Eqs.(4), (7), and (22), i.e., $\phi(0)=0, \phi(1)=1$, $\frac{d \phi(0)}{d \eta}=\frac{d \phi(0)}{d \eta}=0$, and $\phi(1-\eta)=1-\phi(\eta)$. Thus, condition 5 is met.

2n. For the homogeneous states, the sets of constant order parameters for the phase $P_{0} \tilde{\eta}=\hat{\eta}_{0}$ and for phase $\mathrm{P}_{i} \tilde{\eta}=\hat{\eta}_{i}$ should satisfy the thermodynamic equilibrium conditions

$$
X_{i}=-\frac{\partial \psi^{\theta}\left(\theta, \hat{\eta}_{j}\right)}{\partial \eta_{i}}=0, \quad i=1,2, \ldots, n ; \quad j=0,1,2, \ldots, n
$$

for any temperature $\theta$. This condition is already taken into account in Eq.(7).

3n. The free energy should not possess unphysical minima for any temperature. This condition 
is not simple to prove for multiple order parameters, that is why one has to keep potential as simple as possible.

4n. Theory should be invariant with respect to the exchange of phases $\mathrm{P}_{i} \leftrightarrow \mathrm{P}_{j}$ for any $i$ and $j$, including $i=0$ and $j=0$. Also, for some material parameters and temperature, which provide PT $\mathrm{P}_{i} \leftrightarrow \mathrm{P}_{j}$ without involvement of any other phase $\mathrm{P}_{k}$, the description of this $\mathrm{PT}$ should be the same if we choose one of the phase as $P_{0}$.

It is clear that this condition does not have a counterpart for the two-phase system. When we consider the $\mathrm{P}_{i} \leftrightarrow \mathrm{P}_{j} \mathrm{PT}$ alone, we can use the theory for two phases described in the previous Section, in which one of the phases will be chosen as $\mathrm{P}_{0}$. That means that we know all equations for this PT. Condition 4n requires that the same equations should be obtained for this $\mathrm{PT}$ within general $n$-phase theory for phases $\mathrm{P}_{i}$ and $\mathrm{P}_{j}$ for $i \neq 0$ and $j \neq 0$.

As we will see, each $\mathrm{P}_{0} \leftrightarrow \mathrm{P}_{j} \mathrm{PT}$ is described with the help of a single order parameter and does not differ essentially from the two-phase theory (provided that the third phase is not involved). However, the $\mathrm{P}_{i} \leftrightarrow \mathrm{P}_{j} \mathrm{PT}$ involves simultaneous change of two order parameters along some trajectory in the $\eta_{i}-\eta_{j}$ plane, which depends on temperature. In order to make the description of the $\mathrm{P}_{i} \leftrightarrow \mathrm{P}_{j} \mathrm{PT}$ equivalent to the description of the $\mathrm{P}_{0} \leftrightarrow \mathrm{P}_{j} \mathrm{PT}$, this trajectory should be controlled, which includes that under certain material parameters and temperature it is constrained to the straight line connecting $\mathrm{P}_{i}$ and $\mathrm{P}_{j}$.

The key point of the current theory will be an introduction of penalizing terms which executes such a control and do not prevent satisfaction of all desired conditions.

5n. Conditions for thermodynamic instability of homogeneous equilibrium phases that lead to criteria of barrierless PTs between phases $\mathrm{P}_{i}$ and $\mathrm{P}_{j}$ in the general theory for an $n$-phase system should coincide with those for a two-phase system.

Thermodynamic equilibrium state $\hat{\eta}_{j}$ loses its stability when condition

$$
\frac{\partial X_{i}\left(\theta, \hat{\eta}_{j}\right)}{\partial \eta_{k}} \dot{\eta}_{i} \dot{\eta}_{k}=-\frac{\partial^{2} \psi^{\theta}\left(\theta, \hat{\eta}_{j}\right)}{\partial \eta_{i} \partial \eta_{k}} \dot{\eta}_{i} \dot{\eta}_{k} \geq 0
$$

is fulfilled for the first time for some $\dot{\eta}_{i}$. Thus, the instability occurs when $n \times n$ matrix $\partial X_{i} / \partial \eta_{k}$ first ceases to be negative definite or equivalently, $n \times n$ matrix $B_{i k}:=\frac{\partial^{2} \psi^{\theta}\left(\theta, \hat{\eta}_{j}\right)}{\partial \eta_{i} \partial \eta_{k}}$ first ceases to be positive definite. According to Sylvester's criterion, the one of the following conditions 
should be fulfilled for instability of the phase $\hat{\eta}_{j}$ :

$$
\begin{aligned}
& B_{11} \leq 0 ; \quad B_{11} B_{22}-B_{12}^{2} \leq 0 ; \\
& B_{11}\left(B_{22} B_{33}-B_{23}^{2}\right)-B_{12}\left(B_{21} B_{33}-B_{31} B_{23}\right)+B_{33}\left(B_{12} B_{32}-B_{22} B_{31}\right) \leq 0 .
\end{aligned}
$$

In general, it is quite difficult to design a potential for which such sophisticated conditions are reduced to simple conditions for each of the $\mathrm{P}_{0} \leftrightarrow \mathrm{P}_{i}$ or $\mathrm{P}_{j} \leftrightarrow \mathrm{P}_{i}$ transformations, when they are considered separately from other phases. Also, when we considered just two phases, when one of them loses its stability, transformation occurs to another one. In the general case, if, e.g., the third condition Eq.(30) is met, it is not clear to which phase it will transform. Thus, it would be difficult even to compare general results with results for a two-phase case. It is clear that additional simplifications are necessary.

To satisfy condition 5n, we have to assume that if the instability condition Eq. (29) is met for one specific $i$ only, the transformation from the phase $\hat{\eta}_{j}$ will occur toward this $\hat{\eta}_{i}$ phase. Similarly, in the thermodynamic approaches for a sharp interface, transformation conditions from the phase $P_{j}$ to $P_{i}$ are independent of any other phase $P_{k}$ (including $\hat{\eta}_{0}$ ). It is reasonable to assume the same for our PFA. To satisfy condition 5n, we accept the following additional condition.

6n. All mixed second derivatives of $\psi^{\theta}$ with respect to order parameters vanish for any equilibrium phase $\hat{\eta}_{j}$ :

$$
\frac{\partial X_{i}\left(\theta, \hat{\eta}_{j}\right)}{\partial \eta_{k}}=\frac{\partial^{2} \psi^{\theta}\left(\theta, \hat{\eta}_{j}\right)}{\partial \eta_{i} \partial \eta_{k}}=0 \quad \forall k \neq i .
$$

In this case, the instability conditions Eqs.(29) or (30) reduce to

$$
-\frac{\partial X_{i}\left(\theta, \hat{\eta}_{j}\right)}{\partial \eta_{i}}=\frac{\partial^{2} \psi^{\theta}\left(\theta, \hat{\eta}_{j}\right)}{\partial \eta_{i}^{2}} \leq 0 .
$$

Condition 6n significantly simplifies the instability criteria and allows one to analyze them and apply them to the choice of the specific expression for $\psi^{\theta}\left(\theta, \tilde{\eta}_{j}\right)$. Also, it leads to a much simpler expression for this function, for which one can determine all material parameters and have much more confidence that some artificial minima are absent. Thus, the transformation 
conditions between phases read as

$$
\begin{array}{ll}
\mathrm{P}_{0} \rightarrow \mathrm{P}_{i}: & -\frac{\partial X_{i}\left(\theta, \hat{\eta}_{0}\right)}{\partial \eta_{i}}=\frac{\partial^{2} \psi^{\theta}\left(\theta, \hat{\eta}_{0}\right)}{\partial \eta_{i}^{2}} \leq 0 ; \\
\mathrm{P}_{i} \rightarrow \mathrm{P}_{0}: & -\frac{\partial X_{i}\left(\theta, \hat{\eta}_{i}\right)}{\partial \eta_{i}}=\frac{\partial^{2} \psi^{\theta}\left(\theta, \hat{\eta}_{i}\right)}{\partial \eta_{i}^{2}} \leq 0 . \\
\mathrm{P}_{j} \rightarrow \mathrm{P}_{i}: & -\frac{\partial X_{i}\left(\theta, \hat{\eta}_{j}\right)}{\partial \eta_{i}}=\frac{\partial^{2} \psi^{\theta}\left(\theta, \hat{\eta}_{j}\right)}{\partial \eta_{i}^{2}} \leq 0 ; \\
\mathrm{P}_{i} \rightarrow \mathrm{P}_{j}: & -\frac{\partial X_{j}\left(\theta, \hat{\eta}_{i}\right)}{\partial \eta_{j}}=\frac{\partial^{2} \psi^{\theta}\left(\theta, \hat{\eta}_{i}\right)}{\partial \eta_{j}^{2}} \leq 0 .
\end{array}
$$

Note that conditions (33) are valid when any third phase $P_{k}$ is excluded from consideration.

That is why conditions (34) should also be satisfied when the third phase $\mathrm{P}_{k}$ does not appear during $\mathrm{P}_{j} \leftrightarrow \mathrm{P}_{i}$ transformations, i.e., when corresponding constraint on the trajectory connecting $\mathrm{P}_{j}$ and $\mathrm{P}_{i}$ is imposed.

\subsection{Multiphase model}

The simplest expression for the local free energy $\psi^{\theta}$ that includes all of what we derived for a single order-parameter theory and can satisfy all the desired conditions is accepted in the following form:

$$
\begin{aligned}
\psi^{\theta} & =\breve{\psi}^{\theta}+\tilde{\psi}^{\theta}+\psi_{p} \\
\breve{\psi}^{\theta} & =\sum A_{i}(\theta) \eta_{i}^{2}\left(1-\eta_{i}\right)^{2}+\sum \bar{A}_{i j} \eta_{i}^{2} \eta_{j}^{2} ; \quad \bar{A}_{i i}=0 \\
\tilde{\psi}^{\theta} & =\psi_{0}^{\theta}(\theta)+\sum \Delta \psi_{i}^{\theta}(\theta) \phi\left(\eta_{i}\right) ; \quad \Delta \psi_{i}^{\theta}=\psi_{i}^{\theta}-\psi_{0}^{\theta} ; \\
\psi_{p} & =\sum K_{i j}\left(\eta_{i}+\eta_{j}-1\right)^{2} \eta_{i}^{l} \eta_{j}^{l}+\sum K_{i j k} \eta_{i}^{2} \eta_{j}^{2} \eta_{k}^{2} ; l \geq 2 ; \quad K_{i i}=K_{i i k}=K_{i k k}=K_{i j i}=(38)
\end{aligned}
$$

Here $A_{i}$ is proportional to the magnitude of the double-well barriers between phases $\mathrm{P}_{0}$ and $\mathrm{P}_{i}, \bar{A}_{i j}$ contributes to the magnitude of the double-well barriers between phases $\mathrm{P}_{i}$ and $\mathrm{P}_{j}$, and the term $\psi_{p}$ containing coefficients $K_{i j} \geq 0$ and $K_{i j k} \geq 0$ penalizes deviation of the trajectory of the order parameters in $n$-dimensional space $\eta_{i}$ from some lines and planes. Without $\psi_{p}$, the local part of free energy is much simpler than in $[1,2,8]$ and does not contain complex interaction between phases. The term with $\eta_{i}^{2} \eta_{j}^{2} \eta_{k}^{2}$ is positive for any three nonzero order parameters, i.e., it penalizes the presence of the three phases (excluding $P_{0}$ ) at the same material point. This term gives additional means to control the presence of the third phase within the interface between the two other phases, especially, when it is desired to completely 
exclude it. It also contributes to the energy of triple junctions. For homogeneous states, this term always excludes the presence of the three phases at the same point, because it increases energy compared with a two-phase state. When one wants to study the third phase within the interface between the two other phases [24-26], one can set $K_{i j k}=0$, which will simplify analysis. For homogeneous states, the positive terms in $\breve{\psi}^{\theta}$ and $\tilde{\psi}^{\theta}$ exclude appearance of two and three phases $\mathrm{P}_{i}(i \neq 0)$ at the same point. The first terms in $\psi_{p}$ penalizes deviations from hyperplanes $\eta_{i}=0$ orthogonal to the coordinate axes $\eta_{i}$ in the order parameter space and hyperplanes $\eta_{i}+\eta_{j}=1$ passing trough two phases $\mathrm{P}_{i}$ and $\mathrm{P}_{j}$. The exponent $l$ allows one to control relative contribution of these penalties. Since more than two homogeneous phases, say $\mathrm{P}_{i}$ and $\mathrm{P}_{j}$, are forbidden by other terms, the term $\left(\eta_{i}+\eta_{j}-1\right)^{2}$ penalizes deviation from the straight lines $\eta_{i}+\eta_{j}=1, \eta_{k}=0 \forall k \neq i, j$, connecting phases $\mathrm{P}_{i}$ and $\mathrm{P}_{j}$ within plane $\eta_{i}-\eta_{j}$. The term with $\eta_{i}$ penalizes deviation from the coordinate axes in $\eta_{j}$ space, i.e., from straight lines connecting phases $\mathrm{P}_{0}$ and $\mathrm{P}_{i}$. Thus, evolution of $\eta_{i}$ for $K_{i j} \rightarrow \infty$ (at least approximately for large enough $K_{i j}$ ) is constrained to occur along the desired transformation paths. Note that we do not need to use additional constraints to impose evolution of $\eta_{i}$ along the coordinate axes, because for the chosen potential even without them PTs between phases $\mathrm{P}_{0}$ and $\mathrm{P}_{i}$ occur along the straight line connecting these phases. However, without the multiplier $\eta_{i}^{l} \eta_{j}^{l}$, the first term in $\psi_{p}$ will artificially penalize free energy along the coordinate axes in $\eta_{i}$ space and spoil the thermodynamic potential.

For $\mathrm{P}_{0} \leftrightarrow \mathrm{P}_{i}$ PTs described by a single order parameter $\eta_{i}, \psi_{p}$ and the second term in $\breve{\psi}^{\theta}$ disappear and Eqs.(25)-(26) and (35)-(37) reduce to Eqs.(1)-(2) and (24) for two-phase system.

\subsection{Thermodynamic instability conditions}

Direct application of the instability conditions (33)-(34) to free energy (35)-(38) for thermodynamically equilibrium homogeneous phases produces the following PT criteria:

$$
\begin{gathered}
\mathrm{P}_{0} \rightarrow \mathrm{P}_{i}: \quad \frac{\partial^{2} \psi^{\theta}\left(\theta, \hat{\eta}_{0}\right)}{\partial \eta_{i}^{2}} \leq 0 \rightarrow-\Delta \psi_{i}^{\theta} \geq A_{i}(\theta) / 3 ; \\
\mathrm{P}_{i} \rightarrow \mathrm{P}_{0}: \quad \frac{\partial^{2} \psi^{\theta}\left(\theta, \hat{\eta}_{i}\right)}{\partial \eta_{i}^{2}} \leq 0 \rightarrow-\Delta \psi_{i}^{\theta} \leq-A_{i}(\theta) / 3 ; \\
\mathrm{P}_{j} \rightarrow \mathrm{P}_{i}: \quad \frac{\partial^{2} \psi^{\theta}\left(\theta, \hat{\eta}_{j}\right)}{\partial \eta_{i}^{2}} \leq 0 \rightarrow-\Delta \psi_{i}^{\theta} \geq\left(A_{i}(\theta)+\bar{A}\right) / 3 \Rightarrow \text { wrong; } \\
\mathrm{P}_{i} \rightarrow \mathrm{P}_{j}: \quad \frac{\partial^{2} \psi^{\theta}\left(\theta, \hat{\eta}_{i}\right)}{\partial \eta_{j}^{2}} \leq 0 \rightarrow-\Delta \psi_{j}^{\theta} \geq\left(A_{j}(\theta)+\bar{A}\right) / 3 \Rightarrow \text { wrong. }
\end{gathered}
$$


Criteria for $\mathrm{P}_{0} \leftrightarrow \mathrm{P}_{i}$ PTs coincide with PT criteria (19) for the two-phase system, i.e. they satisfy condition $4 \mathrm{n}$. In contrast, conditions for $\mathrm{P}_{i} \leftrightarrow \mathrm{P}_{j}$ PTs are contradictory and do not meet condition $4 \mathrm{n}$. Indeed, they do not depend on the difference in energy between phases $\mathrm{P}_{i}$ and $\mathrm{P}_{j}$ and both barriers $A_{i}$ and $A_{j}$, and depend on the energy of phase $\mathrm{P}_{0}$ (which does not participate in this PT). In addition, since the first and second derivatives of $\psi_{p}$ vanish for all equilibrium phases $\mathrm{P}_{i}$, the term $\psi_{p}$ does not alter phase equilibrium conditions and PT criteria for homogeneous phases. Still, we will demonstrate below that this term is a key player in the development of the consistent PFA for a multiphase system, namely, in making the equations for $\mathrm{P}_{i} \leftrightarrow \mathrm{P}_{j}$ PTs fully equivalent to equations for $\mathrm{P}_{0} \leftrightarrow \mathrm{P}_{i}$ PTs.

\subsection{Constrained model for $\mathrm{P}_{i} \leftrightarrow \mathrm{P}_{j}$ transformations}

We increase parameters $K_{i j}$ (and, if required, $K_{i j k}$ ) to very high values (ideally $K_{i j} \rightarrow \infty$ ) so that they impose constraints $\eta_{i}+\eta_{j}=1$ and $\eta_{k}=0 \forall k \neq i, j$ with any required accuracy. Implementing these constraints in Eqs.(25)-(26) and (35)-(37), we express them in terms of the single order parameter $\eta_{i}$ :

$$
\begin{aligned}
& \breve{\psi}^{\theta}=A_{i j}(\theta) \eta_{i}^{2}\left(1-\eta_{i}\right)^{2} ; \quad A_{i j}=A_{i}+A_{j}+\bar{A}_{i j}=A_{j i} ; \\
& \tilde{\psi}^{\theta}=\Delta \psi_{j}^{\theta}+\Delta \psi_{i j}^{\theta}(\theta) \phi\left(\eta_{i}\right) ; \quad \Delta \psi_{i j}^{\theta}=\Delta \psi_{i}^{\theta}-\Delta \psi_{j}^{\theta} \\
& \psi^{\nabla}=0.5 b_{i j}\left|\nabla \eta_{i}\right|^{2} ; \quad b_{i j}=\beta_{i i}+\beta_{j j}-2 \beta_{i j}=b_{j i}>0 \\
& \dot{\eta}_{i}=l_{i j}\left(-\frac{\partial \psi}{\partial \eta_{i}}+b_{i j} \nabla^{2} \eta_{i}\right) ; \quad l_{i j}=\left(L_{i i} L_{j j}-L_{i j}^{2}\right) /\left(L_{j j}+L_{i j}\right)=l_{i j}>0 .
\end{aligned}
$$

Thermodynamic instability conditions look like

$\mathrm{P}_{j} \rightarrow \mathrm{P}_{i}: \quad-\Delta \psi_{i j}^{\theta} \geq A_{i j}(\theta) / 3 ; \quad \mathrm{P}_{i} \rightarrow \mathrm{P}_{j}: \quad-\Delta \psi_{j i}^{\theta} \geq A_{j i}(\theta) / 3 \rightarrow-\Delta \psi_{i j}^{\theta} \leq-A_{i j}(\theta) / 3 .(45$

It is clear that Eqs.(42)-(45) for $\mathrm{P}_{j} \rightarrow \mathrm{P}_{i}$ PTs coincide to within constants and designations with equations for two-phase system (1)-(2) and (24) and consequently for $\mathrm{P}_{0} \rightarrow \mathrm{P}_{i}$ PTs, as it was required in condition $4 \mathrm{n}$. Note that we explicitly took into account condition (22), without which Eqs.(42)-(44) will not reduce to the two-phase model.

Let us assume similarly to the two-phase model that $\Delta \psi_{i}^{\theta}=-\Delta s_{i}\left(\theta-\theta_{e}^{i}\right)$, where $\Delta s_{i}=$ $s_{i}-s_{0}$ is the jump in entropy between phases $\mathrm{P}_{i}$ and $\mathrm{P}_{0}$ and $\theta_{e}^{i}$ is the thermodynamic equilibrium temperature for phases $\mathrm{P}_{i}$ and $\mathrm{P}_{0}$. Then $\Delta \psi_{j i}^{\theta}=\Delta \psi_{j}^{\theta}-\Delta \psi_{i}^{\theta}=-\Delta s_{j}\left(\theta-\theta_{e}^{j}\right)+\Delta s_{i}\left(\theta-\theta_{e}^{i}\right)=$ $-\Delta s_{j i}\left(\theta-\theta_{e}^{j i}\right)$, where $\Delta s_{j i}=\Delta s_{j}-\Delta s_{i}$ and $\theta_{e}^{j i}=\left(\Delta s_{j} \theta_{e}^{j}-\Delta s_{i} \theta_{e}^{i}\right) / \Delta s_{j i}$. Also, let $A_{i}(\theta)=$ 
$A_{*}^{i} \theta-B_{*}$ and $A_{j i}(\theta)=\tilde{A}_{j i}(\theta)+A_{*}^{j i} \theta-B_{*}^{j i}$, where $\tilde{A}_{j i}$ is some function of temperature. For

phases $\mathrm{P}_{i}$ with different thermal properties we can put $\tilde{A}_{j i}=0$ without loss of generality, like for any $\mathrm{P}_{0}-\mathrm{P}_{i}$ and $\mathrm{P}_{j}-\mathrm{P}_{i} \mathrm{PTs}$. Then, the instability conditions Eqs.(39) and (45) transform to

$$
\begin{array}{lll}
\mathrm{P}_{0} \rightarrow \mathrm{P}_{i}: & \theta<\theta_{c}^{i 0} ; & \theta_{c}^{i 0}:=\left(3 \Delta s_{i} \theta_{e}^{i}-B_{*}^{i 0}\right) /\left(3 \Delta s_{i}-A_{*}^{i}\right) ; \\
\mathrm{P}_{i} \rightarrow \mathrm{P}_{0}: & \theta>\theta_{c}^{i 0} ; & \theta_{c}^{0 i}:=\left(3 \Delta s_{i} \theta_{e}^{i}+B_{*}^{i 0}\right) /\left(3 \Delta s_{i}+A_{*}^{i}\right) ; \\
\mathrm{P}_{i} \rightarrow \mathrm{P}_{j}: & \theta<\theta_{c}^{j i} ; & \theta_{c}^{j i}:=\left(3 \Delta s_{j i} \theta_{e}^{j i}-B_{*}^{j i}\right) /\left(3 \Delta s_{j i}-A_{*}^{j i}\right) ; \\
\mathrm{P}_{j} \rightarrow \mathrm{P}_{i}: & \theta>\theta_{c}^{j i} ; & \theta_{c}^{i j}:=\left(3 \Delta s_{j i} \theta_{e}^{j i}+B_{*}^{j i}\right) /\left(3 \Delta s_{j i}+A_{*}^{j i}\right),
\end{array}
$$

where $\theta_{c}^{j i}$ and $\theta_{c}^{i j}$ are the critical temperatures for barrierless $\mathrm{P}_{i} \rightarrow \mathrm{P}_{j}$ and $\mathrm{P}_{j} \rightarrow \mathrm{P}_{i}$ PTs, including $i=0$ or $j=0$. For the case when $\theta_{e}^{i}=0.5\left(\theta_{c}^{i 0}+\theta_{c}^{0 i}\right)$ and $\theta_{e}^{j i}=0.5\left(\theta_{c}^{j i}+\theta_{c}^{i j}\right)$ one has $A_{*}^{i}=0$ and $A_{*}^{j i}=0$ (i.e., $A_{i}, A_{j i}$ are temperature independent), and

$$
\begin{aligned}
& \theta_{c}^{i 0}:=\theta_{e}^{i}-B_{*}^{i 0} /\left(3 \Delta s_{i}\right) ; \quad \theta_{c}^{0 i}:=\theta_{e}^{i}+B_{*}^{i 0} /\left(3 \Delta s_{i}\right) ; \\
& \theta_{c}^{j i}:=\theta_{e}^{j i}-B_{*}^{j i} /\left(3 \Delta s_{j i}\right) ; \quad \theta_{c}^{i j}:=\theta_{e}^{j i}+B_{*}^{j i} /\left(3 \Delta s_{j i}\right) .
\end{aligned}
$$

In the next subsection, it will be shown that this choice of parameters causes the interface energy and width to be temperature independent.

For phases with equal thermal properties, like martensitic variants or twins, critical temperature does not exists and we accept $A_{j i}(\theta)=\tilde{A}_{j i}(\theta) \neq 0$. We will focus in this paper on phases with different thermal properties.

It is necessary to stress that the PT criteria (45) are valid in the limit $K_{i j} \rightarrow \infty$ only. Analysis of the instability conditions for finite $K_{i j}$ can be found in [32]. In particular, in a very rare case when, for phase $\mathrm{P}_{j}$, the wrong instability criterion (40) is satisfied but correct criterion (45) is not, the local energy minimum slightly shifts from the point $\hat{\eta}_{j}$ along the diagonal $\mathrm{P}_{j} \mathrm{P}_{i}$. This has little effect on the accuracy of simulations. PT to $\mathrm{P}_{i}$ occurs when the correct instability criterion (45) is met only.

\subsection{Analytical Solution}

In contrast to the other multiphase models [1-15], in the developed model each of the PTs for large $K_{i j}$ can be described by a single order parameter without explicit constraints. It allows us to utilize analytical solutions [9] for the interface between two phases propagating in the $x$-direction, including its profile, width $\delta$, and velocity $c$. Due to equivalence of all equations 
for $\mathrm{P}_{0} \leftrightarrow \mathrm{P}_{i}$ and $\mathrm{P}_{j} \leftrightarrow \mathrm{P}_{i} \mathrm{PTs}$, the analytical solution for a propagating with velocity $c$ interface [9] for the $\mathrm{P}_{i} \mathrm{P}_{j}$ interface solutions are:

$$
\eta_{j i}=0.5 \tanh \left[3\left(x-c_{j i} t\right) / \delta_{j i}\right]+0.5 ; \quad \delta_{j i}=\sqrt{18 \beta_{j i} / A_{j i}(\theta)} ; \quad c_{j i}=L_{j i} \delta_{j i} \Delta \psi_{j i}^{\theta}(\theta)
$$

where $\eta_{j i}=\eta_{i}$ for the $i \neq j=0$ for the $\mathrm{P}_{0} \mathrm{P}_{i}$ interface or $\eta_{j i}=\eta_{i}$ at $\eta_{j}=1-\eta_{i}$ for the $\mathrm{P}_{i} \mathrm{P}_{j}$ interface, and $\delta_{j i}$ is defined as a distance between points with $\eta_{i}=0.05$ and $\eta_{i}=0.95$. The energy of the nonequilibrium $S M$ and $S S$ interfaces is defined as an excess energy, with respect to bulk phases, assuming that the Gibbs dividing surface is located where $\eta_{i}=0.5$. This follows from the symmetry of the theory with respect to exchange of any two phases. In a more general case, when such a symmetry is not present, a more general approach for determining the Gibbs dividing surface is justified in [34,35]. Thus,

$$
E^{21}=\int_{-\infty}^{x_{21}^{*}}\left(\psi-\psi_{1}\right) d x+\int_{x_{21}^{*}}^{\infty}\left(\psi-\psi_{2}\right) d x ; \quad E^{s 0}=\int_{-\infty}^{x_{s 0}^{*}}\left(\psi-\psi_{0}\right) d x+\int_{x_{s 0}^{*}}^{\infty}\left(\psi-\psi_{s}\right) d x
$$

Here, $x_{21}^{*}$ is the locations where $\eta_{1}=\eta_{2}=0.5$ for the $\mathrm{P}_{1} \mathrm{P}_{2}$ interface and $x_{s 0}^{*}$ is defined where $\eta_{s}=0.5$ for the $\mathrm{P}_{i} \mathrm{P}_{0}$ interface. Calculations result in $E_{j i}=\beta_{j i} / \delta_{j i}$, which is independent of the driving force $\Delta \psi_{j i}^{\theta}$ and, consequently, coincides with the expression for the energy of the equilibrium interface in [9]. For the particular case $A_{*}^{i j}=0$, the interface energies and width became temperature-independent:

$$
E_{j i}=\sqrt{\beta_{j i} B_{*}^{j i} / 18} ; \quad \delta_{j i}=\sqrt{18 \beta_{j i} / B_{*}^{j i}}
$$

Eqs.(51)and Eqs.(53) allow us to calibrate material parameters $\beta_{j i}, B_{*}^{j i}$, and $L_{j i}$ when the interface energy, width, and velocity are known, along with the thermodynamic parameters $\Delta s_{i j}$ and $\theta_{e}^{i j}$. The ratios of $\mathrm{P}_{i} \mathrm{P}_{j}$ to $\mathrm{P}_{i} \mathrm{P}_{0}$ interface energies and widths, $k_{E}^{j i}$ and $k_{\delta}^{j i}$,

$$
k_{E}^{j i}=\frac{E_{j i}}{E_{i 0}}=\sqrt{\frac{\beta_{j i} B_{*}^{j i}}{\beta_{i 0} B_{*}^{i 0}}} ; \quad k_{\delta}^{j i}=\frac{\delta_{j i}}{\delta_{i 0}}=\sqrt{\frac{\beta_{j i} B_{*}^{i 0}}{\beta^{i 0} B_{*}^{j i}}}
$$

play the key role in determining the material response when the phase $\mathrm{P}_{0}$ appears within $\mathrm{P}_{i} \mathrm{P}_{j}$ interface.

\section{Intermediate melt within the $\beta-\delta$ phase interface in HMX crystal}

Parameter identification. In this paper, we consider $I M\left(P_{0}\right)$ in the energetic material HMX 


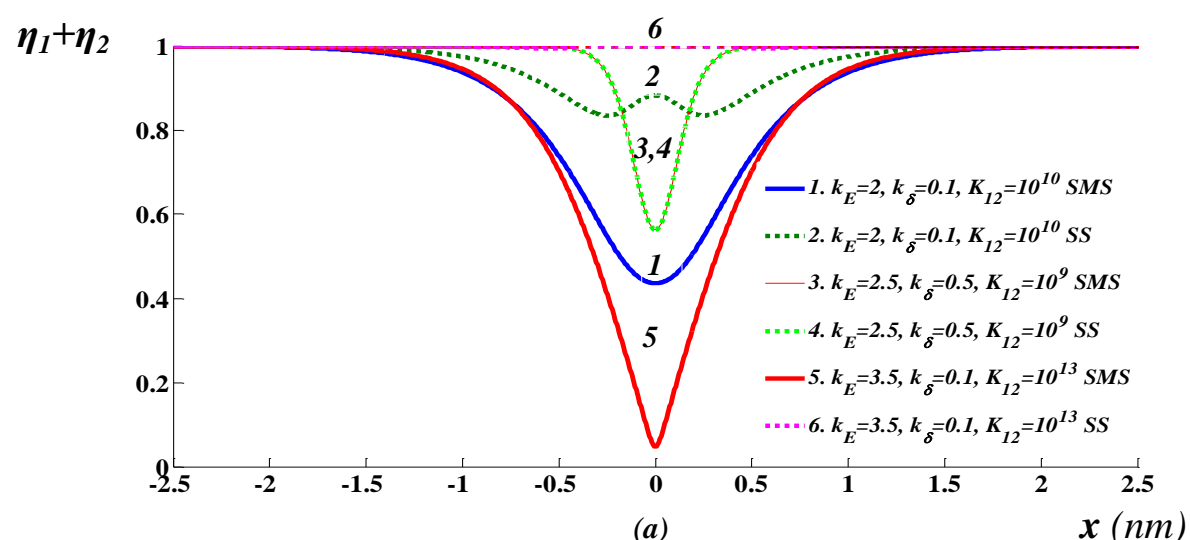

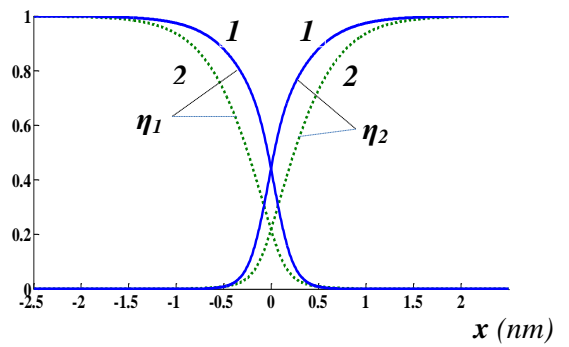

(b)

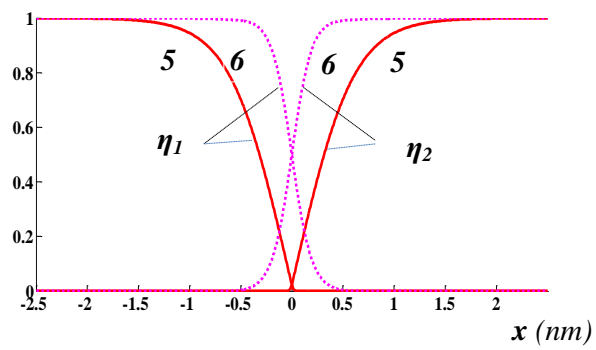

(c)

Figure 1: (a) Stationary distributions of $\eta_{1}+\eta_{2}$ (a), $\eta_{1}$ (b), and $\eta_{2}$ (c) for SMS and SS initial conditions and different values of parameters $K_{12}, k_{E}$ and $k_{\delta}$.

$\left(\mathrm{C}_{4} \mathrm{H}_{8} \mathrm{~N}_{8} \mathrm{O}_{8}\right)$ during a PT between two solid phases, $\delta\left(P_{1}\right)$ and $\beta\left(P_{2}\right)$, which was observed in experiments $[29,30]$. Properties of all three phases are collected in $[24,25,30]$. Thus, in our simulations we use $\Delta s_{10}=-793.79 k J / m^{3} K, \Delta s_{20}=-935.45 k J / m^{3} K$, melting temperatures $\theta_{e}^{10}=550 K$ and $\theta_{e}^{20}=532.14 K ; \theta_{e}^{21}=432 K, L_{10}=L_{20}=2596.5 m^{2} /(N s)$. We also choose $A_{*}^{i}=0$, which corresponds to the temperature-independent interface energies and widths. We choose $E^{21}=1 \mathrm{~J} / \mathrm{m}^{2}$ and $\delta^{21}=1 \mathrm{~nm}$; these values with the help of Eq.(53) result in $\beta_{21}=7.037 \times 10^{-9} \mathrm{~N}$ and $B_{*}^{21}=7.245 \times 10^{6} \mathrm{KJ} / \mathrm{m}^{3}$, and with the help of Eq.(50) in $\theta_{c}^{21}$ and $\theta_{c}^{12}$. Assuming $E^{20}=E^{10}$ and $\delta^{20}=\delta^{10}$, two parameters $\beta_{10}=\beta_{20}$ and $B_{*}^{10}=B_{*}^{20}$ are determined from the chosen values of $k_{E}$ and $k_{\delta}$, which we vary in simulations. In all simulations we use $K_{i j k}=0$ to focus on the effect of $K_{i j}$ on the microstructure.

Numerical procedure. The obtained system of equations has been solved with the help of the finite element code COMSOL for various problems. The numerical procedure reproduced the analytical solution Eq.(51) for each of the three propagating interfaces. Also, it reproduced well (see [32]) quite complex two-variant nanostructure in a NiAl alloy during martensitic PT 
including tip bending and splitting, as it was obtained in simulations in [28] and in experiments in [36]. As we discussed, the theory in [28] for two variants satisfies all required conditions exactly but cannot be generalized for more than two variants.

Simulation results. A 1D $60 \mathrm{~nm}$ long sample was considered with two different initial conditions: (a) equilibrium $S S$ interface and (b) equilibrium $S_{1} M$ and $M S_{2}$ interfaces with quite a broad melt region between the two solids (SMS initial conditions). In Fig. 1 stationary distributions of the order parameter are plotted for $\theta=\theta_{e}^{21}=432 K$ (i.e., $100 \mathrm{~K}$ below $\theta_{e}^{m}$ ), and some specific $k_{E}, k_{\delta}, K_{12}$, for both $S M S$ and $S S$ initial conditions. $S S$ interface corresponds to $\eta_{1}+\eta_{2}=1$ and the intersection point of the curves $\eta_{1}$ and $\eta_{2}$ is $\eta_{1}=\eta_{2}=0.5$. Any deviation from the line $\eta_{1}+\eta_{2}=1$ represents some degree of disordering, i.e., $I M$. Curves 3 and 4 coincide, indicating independence of the stationary state of initial conditions, which is possible for a relatively small magnitude of the energy barrier between solids and melt $K_{12}=10^{9}$. For larger barrier $K_{12}$ and different combinations of $k_{E}$ and $k_{\delta}$, the stationary distribution splits into two solutions from both sides of the energy maximum, one closer to the $S S$ interface and another one with higher disordering and closer to the $S M S$ interface. This is also clearly visible in the $\eta_{1}-\eta_{2}$ plane, when the trajectory corresponding to the stationary solutions for order parameters are superposed into the contour plot of the local free energy (Fig. 2). For a very large barrier, one of the solutions coincides with the $S S$ interface and another one represents two $S M$ interfaces with almost complete melt in the middle. Note that even for $k_{E}=2$, when there is no driving force for melting due to reduction in surface energy, due to relatively small barrier $K_{12}=10^{10}$, there is some disordering for $S S$ initial conditions and significant disordering for $S M S$ initial conditions.

In Figs. 3 and 4 , the minimum value of the parameter $\left(\eta_{1}+\eta_{2}\right)_{m i n}$, which characterizes the degree of disordering, is presented as a function of $k_{\delta}$ and $k_{E}$, respectively, for different values of $k_{E}$ and $k_{\delta}$, respectively, as well as $K_{12}$. Both $S M S$ and $S S$ initial conditions were considered for each case. For a large enough penalizing energy, $K_{12}=10^{13} \mathrm{~J} / \mathrm{m}^{3}$ (Figs. 3 and 4 , f), there are two solutions corresponding to $\left(\eta_{1}+\eta_{2}\right)_{\min }=1(S S$ interface $)$ and small $\left(\eta_{1}+\eta_{2}\right)_{\min }(S M S$ interface with $I M)$, and both solutions are practically independent of variable parameters, $k_{E}$ or $k_{\delta}$, respectively. Even for $k_{E}<1$, when formation of even a single $S M$ interface leads to an energy increase in comparison with the $S S$ interface, if $S M S$ is formed (e.g., at much higher 


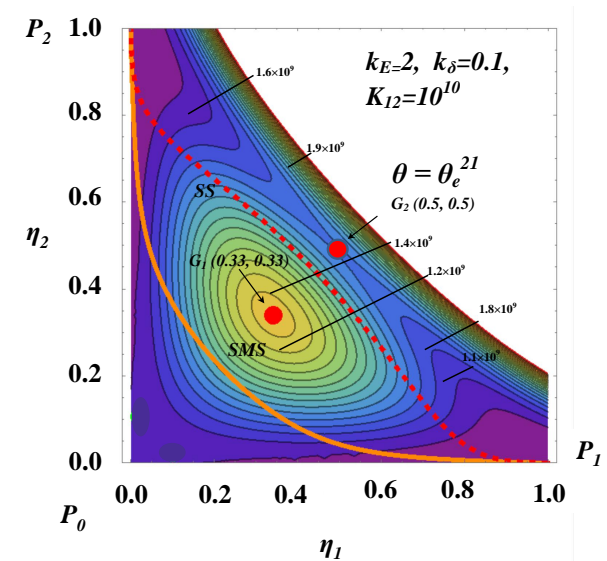

(a)

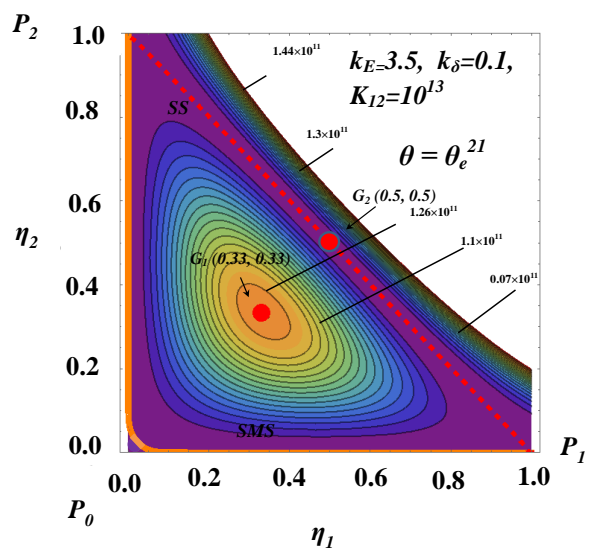

(b)

Figure 2: Contour plot of the local free energy with superposed trajectories corresponding to the stationary solutions for the order parameters in $\eta_{1}-\eta_{2}$ plane for SMS and SS initial conditions and $\theta=\theta_{e}^{21}$. Results are presented for $k_{E}=2, k_{\delta}=0.1$, and $K_{12}=10^{10} \mathrm{~J} / \mathrm{m}^{3}$ (a) and for $k_{E}=3.5, k_{\delta}=0.1$, and $K_{12}=10^{13} \mathrm{~J} / \mathrm{m}^{3}$ (b). Red dots represents some minimaxes of the energy.

temperature and then quenched to $\theta=\theta_{e}^{21}$ ), it retains as a metastable state. This is because of the high energy barrier separating $S M S$ and $S S$ configurations, see Fig. 2.

At the opposite end of our simulations, for small value of $K_{12}=10^{9} \mathrm{~J} / \mathrm{m}^{3}$ (Figs. 3 and 4 , a), there is one solution only independent of the initial conditions. Generally, with increasing $k_{\delta}$, the value of $\left(\eta_{1}+\eta_{2}\right)_{\min }$ reduces, with some small exception for $k_{\delta}<0.3$. With increasing $k_{E}$, the degree of disordering reduces monotonously.

When $K_{12}$ reduces from $10^{13} \mathrm{~J} / \mathrm{m}^{3}$ to $10^{11} \mathrm{~J} / \mathrm{m}^{3}$ and $2 \times 10^{10} \mathrm{~J} / \mathrm{m}^{3}$, the behavior becomes more interesting. There are again two solutions, one with $\left(\eta_{1}+\eta_{2}\right)_{\min } \simeq 1$ and another one with small $\left(\eta_{1}+\eta_{2}\right)_{m i n}$, slightly decreasing with increasing $k_{E}$ and $k_{\delta}$ (Figs. 3 and 4 , d and e). While disordered solutions exist for all $k_{\delta}$, the $S S$ interface ceases to exist at some critical value of $k_{\delta}^{c}$ (the smaller $k_{E}$ is, the larger $k_{\delta}^{c}$ becomes). Above these critical values, there is a jump from $S S$ interface to $S M S$ interface and the unique solution is independent of the initial conditions. When $k_{E}$ is considered as an independent argument (Fig. 4), for $K_{12}=10^{11} \mathrm{~J} / \mathrm{m}^{3}$ and $K_{12}=2 \times 10^{10} \mathrm{~J} / \mathrm{m}^{3}$, behavior is similar to that versus $k_{\delta}$. Namely, while disordered solutions exist for all $k_{E}, S S$ interface ceases to exist at some critical value of $k_{E}^{c}$ (the smaller $k_{\delta}$ is, the larger $k_{E}^{c}$ becomes), and there is a jump to the unique $S M S$ solution. With reduction 

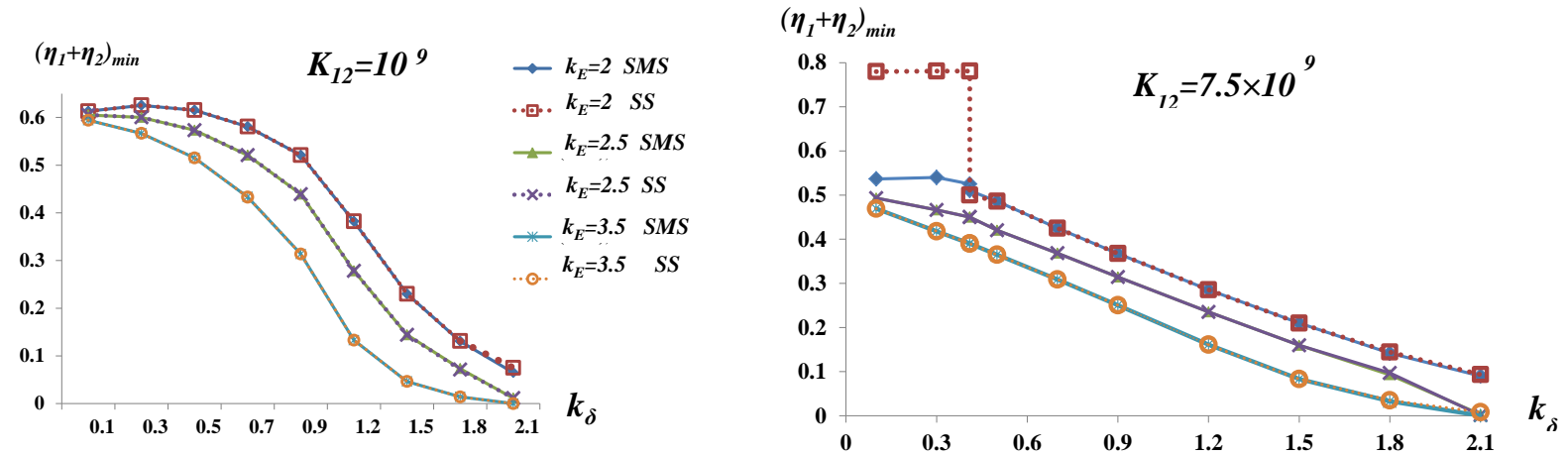

(a)

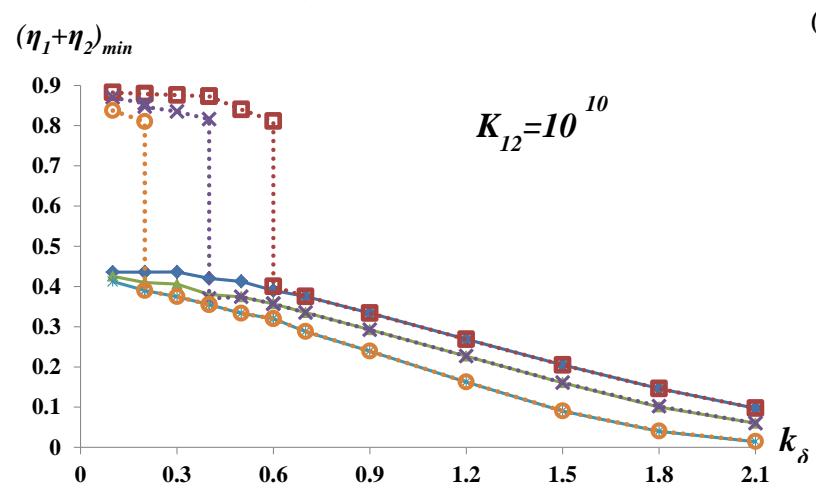

$\left(\eta_{1}+\eta_{2}\right)_{\min }$

(b)

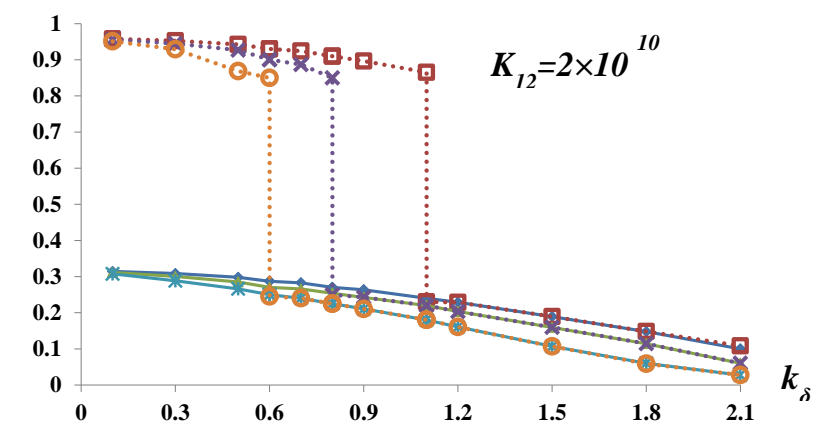

(c)

(d)

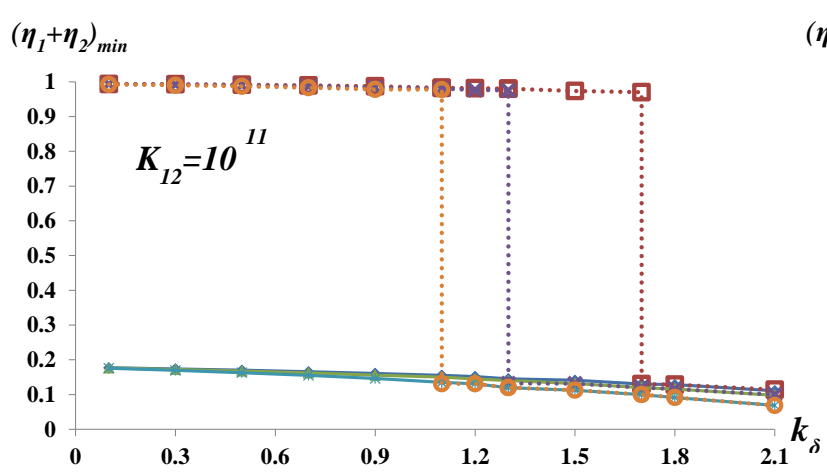

(e)

$\left(\boldsymbol{\eta}_{1}+\boldsymbol{\eta}_{2}\right)_{\min }$

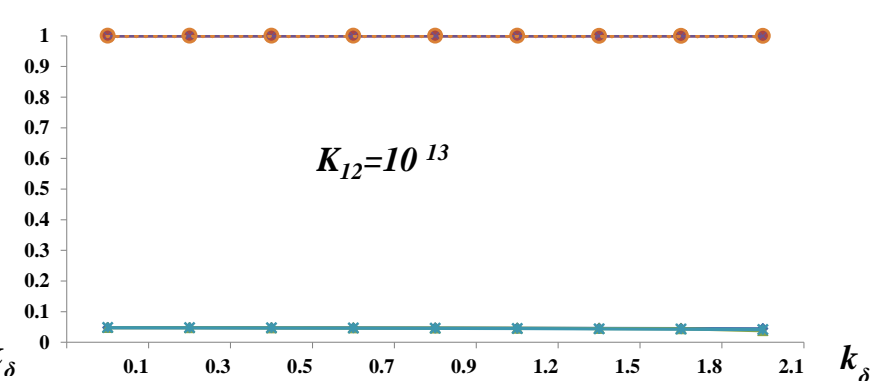

$(f)$

Figure 3: The minimum stationary value of $\left(\eta_{1}+\eta_{2}\right)_{\min }$ versus $k_{\delta}$ for $S M S$ and $S S$ initial conditions and for different $K_{12}$ and $k_{E}$ values. 
$\left(\boldsymbol{\eta}_{1}+\boldsymbol{\eta}_{2}\right)_{\min }$

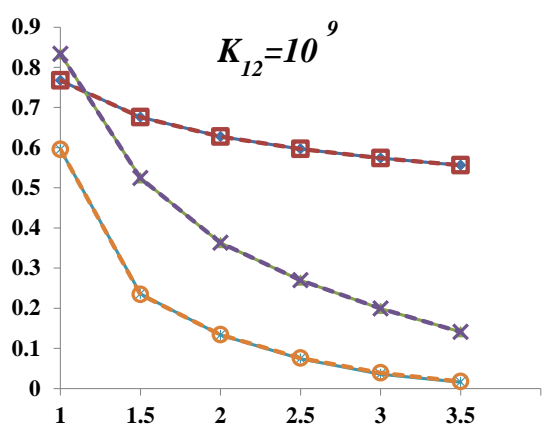

(a)

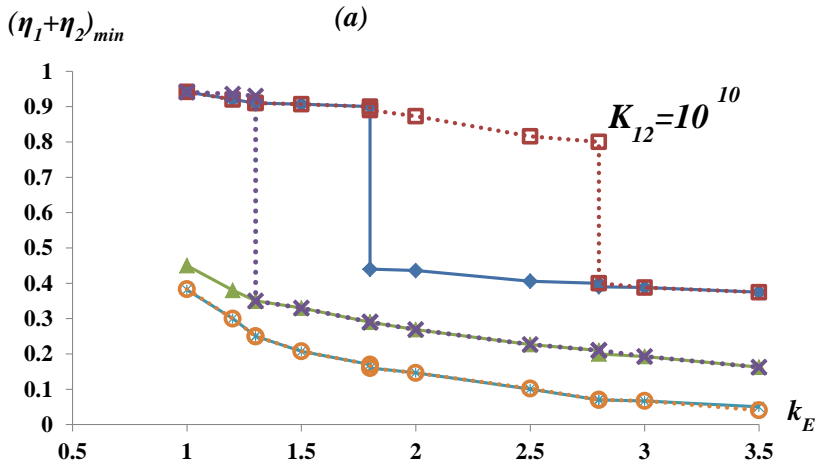

(c)

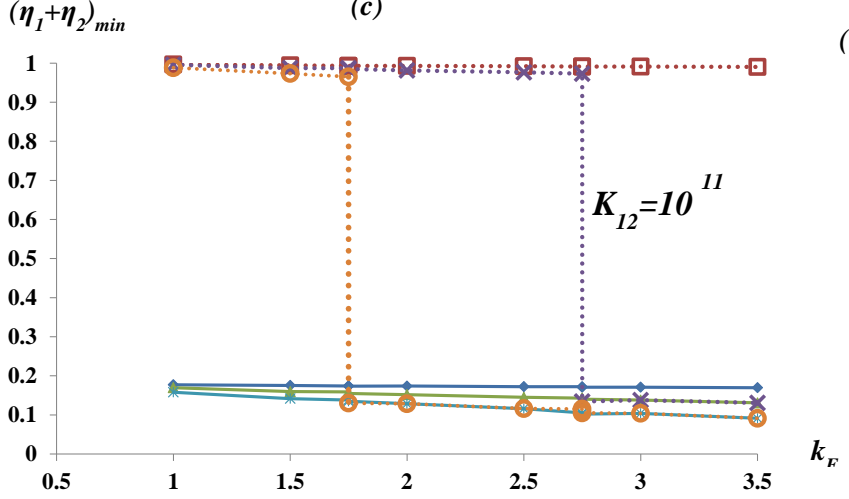

$(e)$

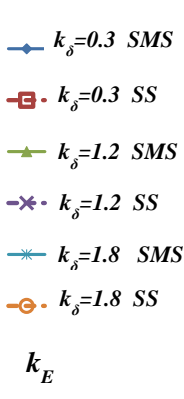

$\left(\boldsymbol{\eta}_{1}+\boldsymbol{\eta}_{2}\right)_{\min }$

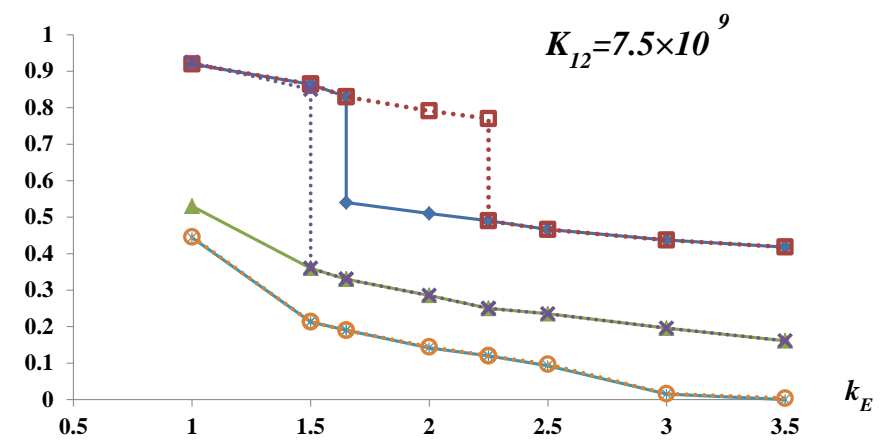

$\left(\eta_{1}+\eta_{2}\right)_{\min }$

(b)

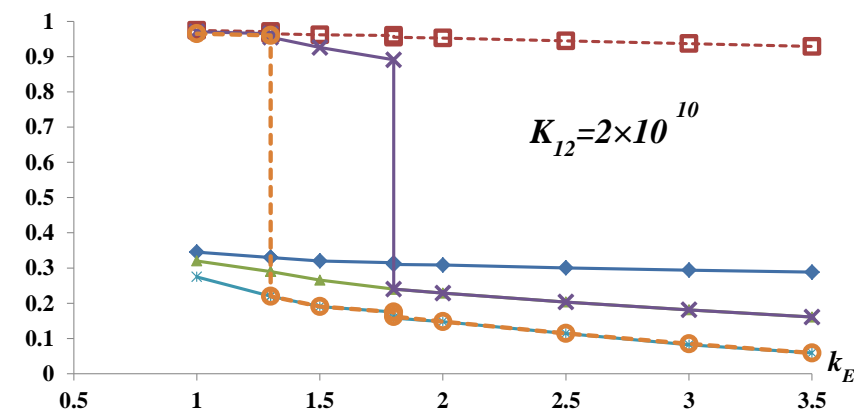

(d)

$\left(\boldsymbol{\eta}_{1}+\boldsymbol{\eta}_{2}\right)_{\min }$

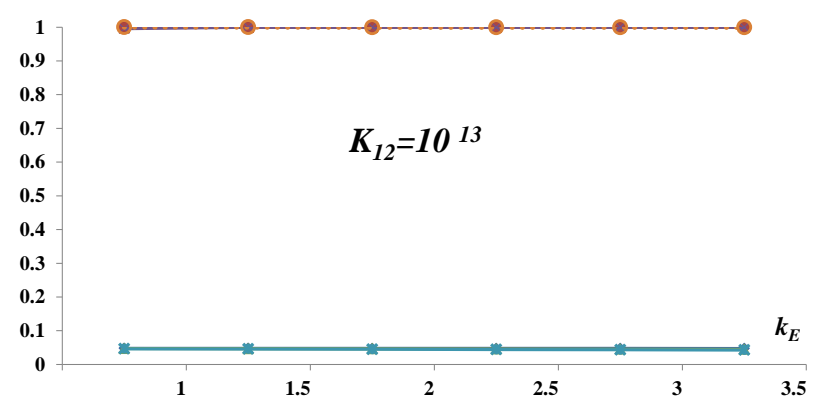

(f)

Figure 4: The minimum stationary value of $\left(\eta_{1}+\eta_{2}\right)_{\min }$ versus $k_{E}$ for $S M S$ and $S S$ initial conditions and for different $K_{12}$ and $k_{\delta}$ values. 
in $K_{12}$, the threshold $k_{E}^{c}$ reduces.

With further reduction in $K_{12}$, Figs. 3 and 4 should be discussed separately. In Fig. 3 the $S M S$ interface exists for all parameters under study. With reduction in $K_{12}$, the critical value of $k_{\delta}^{c}$ reduces and in the coexistence regions curves are becoming more strongly dependent on $k_{\delta}$ and $\left(\eta_{1}+\eta_{2}\right)_{\text {min }}$ more strongly deviates from 1 and 0 . Below some critical $K_{12}^{c}$, the less disordered solution close to $S S$ ceases to exist and the unique solution remains only.

When $k_{E}$ is considered as an independent argument (Fig. 4), for $K_{12}=2 \times 10^{10} \mathrm{~J} / \mathrm{m}^{3}$ and $K_{12}=7.5 \times 10^{9} \mathrm{~J} / \mathrm{m}^{3}$, the $S M S$ solution also ceases to exist below some critical $k_{E *}^{c}$. Thus, there are regions of $k_{E}$, for which only either slightly or strongly disordered interface exists, between which both solutions exist, i.e., there is a hysteresis loop.

Next, we solve problem for propagating the $S M S$ interface for different temperatures for $k_{\delta}=0.1, k_{E}=2$, and several values of $K_{12}$. After the interface profile becomes steady, we determine width of the melt, $\delta^{*}$ as a distance between points with $\eta_{1}=0.5$ and $\eta_{2}=0.5$; it clear that for a perfect $S S$ interface $\delta^{*}=0$. Energy of the $S M S$ interface is defined as

$$
E^{*}=\int_{-\infty}^{x^{*}}\left(\psi-\psi_{1}\right) d x+\int_{x^{*}}^{\infty}\left(\psi-\psi_{2}\right) d x
$$

where $x^{*}$ is the point of intersection of the profiles $\eta_{1}$ and $\eta_{2}$. When temperature is excluded as a parameter, one can obtain dependence of the $S M S$ interface energy $E^{*}$ on the $I M$ width $\delta^{*}$ for different temperatures and $K_{12}$ (Fig. 5). It is clear that with increasing temperature width of the $I M$ increases. Since energy of the $S M S$ interface decreases with increasing temperature for the chosen $k_{\delta}$ and $k_{E}$, energy $E^{*}$ is decreasing as a function of the width of $I M \delta^{*}$ (Fig. 5).

Note that since and when we have two stationary solutions corresponding to the local energy minimum, it should be the third solution corresponding to the minimax of the energy and representing a critical nucleus between these two states. For a different model, such a critical nucleus was treated in [25-27].

\section{Concluding remarks}

In the paper, we formulated and justified the main conditions for the free energy potential for multiphase phase field theory for temperature-induced PTs, and developed a theory, which satisfies all these conditions. The theory does not include any explicit constraint on the order 
parameters. Instead, penalizing terms in the local energy control absence or presence and extent of presence of the third phase within an interface between two other phases. When conditions correspond to the absence of the third phase, the desired PT criteria follow from the thermodynamic instability conditions of the bulk equilibrium phases. The proposed approach resolves a long-standing problem in the PFA. Different approaches in the literature are discussed in terms of the formulated here and some other conditions and it is concluded that they do not satisfy some of them. Formulated equations are implemented in the finite-element code COMSOL and used for studying PTs between $\beta$ and $\delta$ phases of HMX energetic crystal via $I M 100^{\circ} \mathrm{C}$ below melting temperature. A parametric study of these two- and three-phase structures is performed. They consist of either two microstructures, one with smaller (or absent) and one with larger (or complete) disordering within $S S$ interface, or these solutions coincide. For the cases with two solutions, each (or only one) of them may disappear leading to a barrierless jump to the alternative solution. If both solutions may disappear, there is a hysteresis region in which both of them exist. The important point is that even for a very large magnitude of the penalizing term which provides a perfect two-phase interface without the third phase, there is an alternative solution that includes the third phase. Depending on material parameters and temperature, it can be stable or metastable. That is why the requirement about elimination of the third phase within an interface between two other phases should be formulated more carefully. Our approach is more flexible, because we do not require absence of the third phase but the possibility to control it, including elimination. It does not prevent existence of an alternative solution with the third phase, and we can find whether the solution without the third phase is stable, metastable or unstable, and quantify these findings. Stability of the propagating $S S$ and $S M S$ interfaces can be studied using methods developed in $[37]$.

The developed requirements and approach are applicable to various PTs between multiple solid and liquid phases, including reconstructive PTs and twinning in solids, and can be elaborated for stress-induced PTs, electromagnetic PTs, diffusive PTs described by Cahn-Hilliard equations, as well as the evolution of grain structures.

\section{Acknowledgements}


The support of NSF (DMR-1434613 and CMMI-1536925), ARO (W911NF-12-1-0340), DARPA (W31P4Q-13-1-0010), ONR, and Iowa State University is gratefully acknowledged.

\section{Appendix A}

\section{A.1. Comparison with existing potentials}

\section{A.1.1. Single order parameter}

For a single order parameter, the formal theory for the Landau potential of practically arbitrary degree based on group theoretical (symmetry) consideration is presented in $[38,39]$. Analysis, including phase diagrams, was performed in terms of coefficients of Landau potentials. Such potentials exhibit multiple minima corresponding to multiple phases. When PTs between two phases are considered, especially polymorphic PTs in solids, thermodynamic potentials $\psi^{\theta}=\bar{a} \eta^{2}+\bar{b} \eta^{3}+\bar{c} \eta^{4}$ or $\psi^{\theta}=\bar{a} \eta^{2}+\bar{b} \eta^{4}+\bar{c} \eta^{6}$ were used without any general requirements, except that they should have two minima separated by an energy barrier [3-7,38-44]. In most works $\bar{a}=\bar{a}_{0}\left(\theta-\theta_{c}\right)$ was assumed, which defines $\theta_{c}$ as the critical temperature when thermodynamic instability occurs, i.e., energy minimum at $\eta=0$ disappears. The thermodynamic equilibrium value of the order parameter at the second minimum was dependent on the temperature, similar to the continuous second-order PTs. This means that the PT does not have the end point and structural changes occur continuously. The order parameter is assumed to be small, like in Landau theory of the second-order PTs [45], which justifies Taylor expansion for the energy with a limited number of terms. There are no specifically introduced interpolation functions and variation of material properties, like in Eq.(3). Variation of all material properties follows directly from the chosen potential and in many cases they correspond to experiments [40] for the second order and close to the second order PTs.

For the description of the first-order PTs, which have the end point and further structural changes do not occur after completing a PT (like melting and martensitic phase transformations in steels and shape memory alloys), the order parameter should not change after a PT. Then the order parameters for bulk phases can be taken as 0 and 1 . It cannot be considered as a 
small number and higher degrees of $\eta$ make similar contributions as the lower degrees. This condition as well as thermodynamic consistency, which are very similar to conditions 1 and 2 , were formulated and satisfied in $[33,46,47]$ for melting. Since interaction between communities working on melting and martensitic PTs, twinning, and dislocations was very limited, such conditions were not used and satisfied in these fields for a long time, even now (see, e.g., [3-7] for martensitic PT, including twinning, and [48-51] for dislocations). Also, for twinning and dislocations these conditions are related to transformation strain and Burgers vector rather than to change in free energy, which is zero. Conditions 1-4 were formulated and satisfied in $[1,2,8,52,53]$ for martensitic PTs and twinning, and in $[8,54]$ for dislocations, where they were motivated by correctness of the stress-strain curve, which also lead to conditions for the free energy and interpolating functions for all parameters. For melting, the thermodynamic stability condition was imposed (i.e., the pre-factor of a double-well barrier must be positive) $[16,17,33,46,47]$ instead of condition 4 . We believe that the main reason for this is the following. In order to increase interface width by a factor of $k$ without changing interface energy and velocity (see Eq.(51)), one has to increase $\beta$ by a factor of $k$ and reduce $A$ and $L$ by a factor of $k$. Thus, the magnitude of the double well barrier significantly reduces ( $k$ may be as large as 1000) and a correct description of thermodynamic instability and barrierless nucleation is impossible. For such a small double well barrier, thermodynamic instability may occur quite close to the thermodynamic equilibrium temperature, which will lead to artificial barrierless nucleation of a (meta)stable phase within an unstable one. To avoid this, one has to insure satisfaction of the stability condition for any temperature, i.e., instability criterion should not be affected by the thermodynamic driving force. This was done by choosing interpolating function $\bar{\varphi}(\eta)=\eta^{3}\left(10-15 \eta+6 \eta^{2}\right)$, which satisfies all desired conditions $\left(\bar{\varphi}(0)=\bar{\varphi}^{\prime}(0)=\right.$ $\bar{\varphi}^{\prime}(1)=0, \bar{\varphi}(1)=1$, and $\left.\bar{\varphi}(1-\eta)=1-\bar{\varphi}(\eta)\right)$. It also satisfies conditions $\bar{\varphi}^{\prime \prime}(0)=\bar{\varphi}^{\prime \prime}(1)=0$, which eliminates participation of any material parameter or function multiplied by $\bar{\varphi}(\eta)$ from the instability condition. Thus, for the energy

$$
\bar{\psi}^{\theta}(\theta, \eta)=\psi_{0}^{\theta}(\theta)+\Delta \psi^{\theta}(\theta) \bar{\varphi}(\eta)+A \eta^{2}(1-\eta)^{2}
$$

accepted in $[16,17,33]$ instability conditions (12) and (13) reduce to

$$
\mathrm{P}_{0} \rightarrow \mathrm{P}_{1}: \quad \partial^{2} \bar{\psi}^{\theta}(\theta, 0) / \partial \eta^{2}=2 A \leq 0 ; \quad \mathrm{P}_{1} \rightarrow \mathrm{P}_{0}: \quad \partial^{2} \bar{\psi}^{\theta}(\theta, 1) / \partial \eta^{2}=2 A \leq 0
$$


Such instability conditions are contradictory because both phases simultaneously loose their stability. That is why interpolation function (56) is not suitable for our purposes. However, if stability conditions were imposed instead of instability $[16,17,33]$, function $(56)$ is very convenient because the system is stable for $A>0$ independent of the driving force (temperature).

In contrast, the thermodynamic instability was included in consideration in $[1,2,8]$, which resulted in PT criteria. This allows one to consider problems at the actual physical space scale where thermodynamic instability is important, e.g., for very fast heating much higher than the melting and even solid instability temperatures [55,56], as well as for barrierless surface-induced melting, especially for nanoparticles [57,58], and for melting within an interface between two solids [24-26, 29,31], which all may occur significantly below melting and melt instability temperatures. The interpolation function (18) that satisfies all conditions has been used for various applications for a long time $[1,2,33,46,47,52,53]$.

Within even six-degree potential (2-4-6 potential), we obtained [8] that the interpolating function $\varphi_{6}(\eta)=0.5 a \eta^{2}+(3-a) \eta^{4}+0.5(a-4) \eta^{6}$ satisfies conditions 1-4. However, it does not satisfy condition $\varphi_{6}(1-\eta)=1-\varphi_{6}(\eta)$. That means that it cannot be used not only in our multiphase system but also for a two-phase system when both phases are equivalent. The same is true for the function $(8)$ for $a \neq 3$. In particular, it cannot be used for twinning, while cases where $a \neq 3$ were studied exploratory for twinning in [59].

Note that for larger-scale theories one can waive the requirements $\varphi^{\prime}(0)=\bar{\varphi}^{\prime}(1)=0$ for interpolation functions. Thus, it was accepted in $[19,20] \psi^{\theta}(\theta, \eta)=\psi_{0}^{\theta}(\theta)+\Delta \psi^{\theta}(\theta) \eta+A \eta(1-\eta)$, where $0 \leq \eta \leq 1$ was interpreted as the concentration of phase 1 and these inequalities ensured

that $\eta=0$ and $\eta=1$ corresponds to the equilibrium phases while the thermodynamic driving force at these points is not zero. A similar barrier term was used in the double obstacle potential [11] but with a different smooth interpolating function for $\Delta \psi^{\theta}$.

\section{A.2. Multiple order parameter}

Theories without a constraint. They are mostly devoted to the description of multivariant martensitic PTs [3-7]. They are based on the fourth or sixth degree polynomials in terms of order parameters $\eta_{i}$ that describe PTs austenite $\mathrm{A}$ - martensitic variants $\mathrm{M}_{i}$ and satisfy the required symmetry conditions. Since all martensitic variants are symmetry-related and have the same thermal (chemical) free energy, these theories represent a particular case of the gen- 
eral theory for multiphase system. None of the above requirements to the free energy were imposed and met in [3-7], i.e., thermodynamic equilibrium order parameter for each $\mathrm{M}_{i} \eta_{i} \neq 1$ and depends on temperature (and stresses), thermodynamic instability conditions were not considered, and PTs $\mathrm{M}_{j} \leftrightarrow \mathrm{M}_{i}$ occurred along some temperature dependent path within the $\eta_{i}-\eta_{j}$ plane. No specific interpolating function for material properties have been introduced, i.e., they directly follow from the chosen polynomial. This is similar to the description of the second-order and close to the second order continuous PTs in [40] but was applied to the strongly first-order PTs. Also, matrixes $L_{i j}$ and $\beta_{i j}$ are reduced to the unit matrix multiplied by a scalar. Since theories [3-7] possess minima corresponding to A and all martensitic variants $\mathrm{M}_{i}$, they reproduce evolution of complex multivariant microstructure. However, it could not quantitatively correspond to a chosen specific material because material properties were not properly interpolated between their values in the bulk phases and thermodynamic equilibrium order parameters were not constant but depended on temperature (and stress tensor). Conditions close to 1-4 and 1n-6n have been formulated and satisfied in $[1,2,8]$, but without condition 5, i.e., still PTs $\mathrm{M}_{j} \leftrightarrow \mathrm{M}_{i}$ were not properly described and parameters of $\mathrm{M}_{j}-\mathrm{M}_{i}$ interfaces could not be properly calibrated and controlled. Matrix form of $\beta_{i j}$, i.e., additional material parameters, have been introduced in [60,61], which in particular allowed us to introduce and study the effect of the energy of the $\mathrm{M}_{j} \leftrightarrow \mathrm{M}_{i}$ interface independent of the energy of A- $\mathrm{M}_{i}$ interfaces.

Theories with hyperspherical order parameters. In order to describe $\mathrm{M}_{j} \leftrightarrow \mathrm{M}_{i} \mathrm{PTs}$ in the same way as $\mathrm{A} \leftrightarrow \mathrm{M}_{i}$ PTs, a thermodynamic potential in hyperspherical order parameters was developed [8], in which $A$ is at the center of the sphere, and all martensitic variants $M_{i}$ are located at the hypersphere. Belonging to the hypersphere represents a nonlinear constraint. Because of impossibility to satisfy some of the requirements, namely to obtain consistent PT criteria from the thermodynamic instability conditions, the nonlinear constraint for the hyperspherical order parameters was substituted in [28] with the linear constraint of the type $\sum \eta_{i}=1$, which, however, does not include A. Still, PT criteria could not be obtained in a consistent way for more than three phases. For three phases the constraint is linear for both models [8] and [28] and in polar order parameters this theory is completely consistent with the two-phase theory and produces proper PT criteria. It was generalized for three arbitrary phases in [24-26]. 
Thus, theory in [24-26] is currently the only theory that satisfies exactly all requirements for three-phase material. Due to polar order parameters, it does not need to satisfy condition 5 , that is why it can utilize interpolation function Eq.(8) with arbitrary $0 \leq a \leq 6$ for each pair of phases. However, we fail to generalize it for more than three phases. In this case, constraint should be used which does not allow one to derive consistent PT criteria from thermodynamic instability conditions.

Theories with a constraint. Traditional multiphase theories [9-18] include constraint $\sum \eta_{i}=1$ applied to all phases. It can be explicitly excluded for two phases only, in contrast to the case with three phases for polar order parameters $[8,24-26]$. This means that the problem to derive consistent PT criteria from thermodynamic instability conditions exists even for three phases. The first theory [9] does not make special efforts that the PT between any two phases occurs along the fixed line in the order parameter space. That is why the third phase may appear at the interface between two phases. This does not allow one to use an analytical solution to calibrate material parameters in terms of interface energy, mobility, as well as width (if kept physical rather than computational). Also, potential in [9] does not include products of more than two order parameters, which however, is easy to add. However, the main basic problem in [9] is that the derivation of the kinetic equations includes a simplifying assumption, which makes theory thermodynamically inconsistent, i.e., the second law of thermodynamics is not strictly imposed. As it is shown in [21], the solution of the Ginzburg-Landau equations from [9] lead, in some cases, to the energy increase.

If a computational interface width is much larger than the actual one, presence of the third phase contributes to the width-dependence of the solution, which is desirable to avoid. That is why in $[16,17]$ equations are derived in a way enforcing that PTs always occur along the straight line connecting two phases. With the choice of an interpolating function generalizing $\bar{\varphi}(\eta)$ for multiple phases, all bulk phases are stable or metastable independent of the driving force or temperature. It is not clear how to generalize theory for more than three phases. Also, kinetic coefficients must be scalar and equal for all PTs. While in [9] constraint was imposed by excluding one of the order parameters, in $[10,16,17]$ the method of Lagrangian multipliers was used. When a Lagrangian multiplier was used, the Ginzburg-Landau equations for $n$ phases 
take the form

$$
\dot{\eta}_{i}=L\left(X_{i}-\Lambda\right)=L\left(-\frac{\delta \psi}{\delta \eta_{i}}-\Lambda\right)
$$

Adding all equations and using constraint, we obtain $\Lambda=\sum X_{j} / n$ and substituting it in Eq.(58) it transforms to

$$
\dot{\eta}_{i}=L\left(X_{i}-\sum X_{j} / n\right)=L_{i j} X_{j} ; \quad L_{i j}=L_{j i}=L\left(\delta_{i j}^{K}-U_{i j} / n\right)
$$

where $\delta_{i j}^{K}$ is the Kronecker delta and all components of the matrix $U_{i j}$ are equal to one. With such a matrix $L_{i j}$, constraint $\sum \dot{\eta}_{i}=0$ is fulfilled automatically. It was, however, stated in [18] that the use of Lagrangian multiplier method gives results different from direct exclusion of one of the order parameters, even for two phases. Let us analyze this statement for two order parameters obeying constraint $\eta_{1}+\eta_{2}=1$. The dissipation rate is $D_{1}=X_{1} \dot{\eta}_{1}+X_{2} \dot{\eta}_{2}=\left(X_{1}-\right.$ $\left.X_{2}\right) \dot{\eta}_{1}$ when $\eta_{2}$ is directly excluded and $D_{2}=X_{1} \dot{\eta}_{1}+X_{2} \dot{\eta}_{2}-\Lambda\left(\dot{\eta}_{1}+\dot{\eta}_{2}\right)=\left(X_{1}-\Lambda\right) \dot{\eta}_{1}+\left(X_{2}-\Lambda\right) \dot{\eta}_{2}$ when a Lagrangian multiplier is used. Then, using the same kinetic coefficient, the linear relationship between thermodynamic forces and rates for both cases are:

$$
\dot{\eta}_{1}=L\left(X_{1}-X_{2}\right) ; \quad \dot{\eta}_{1}=L\left(X_{1}-\Lambda\right)=L\left(X_{1}-X_{2}\right) / 2
$$

which lead to the conclusion that "the Lagrange multiplier approach does not reduce to the single phase formulation," see [18]. The main reason for this discrepancy is that the thermodynamic forces and rates in different representations should not be connected by the same kinetic coefficient. If, e.g., in Eq. $(60)_{2}$ we would choose the kinetic coefficient $L_{2}$ different from $L$ in Eq. $(60)_{1}$, we would not have a problem, and from the equivalence of both kinetic equations we can conclude that $L=L_{2} / 2$. This becomes more clear if we substitute the expression for $\Lambda=\left(X_{1}+X_{2}\right) / 2$ in $D_{2}: D_{2}=0.5\left(X_{1}-X_{2}\right) \dot{\eta}_{1}-0.5\left(X_{1}-X_{2}\right) \dot{\eta}_{2}$. It is evident that using constraint we obtain $D_{1}=D_{2}$. However, thermodynamic force $0.5\left(X_{1}-X_{2}\right)$ cannot be connected to $\dot{\eta}_{1}$ by the same kinetic coefficient as $\left(X_{1}-X_{2}\right)$. For multiple order parameters, even if the force obtained by direct exclusion of one of the order parameters can be connected to the conjugate rate using a single scalar, one may need a matrix connection between all forces and rates for the Lagrangian multiplier method to obtain an equivalent result. The primary expressions for the thermodynamic forces and rates should be taken from the expression for the dissipation rate expressed in terms of independent rates, i.e., after direct exclusion of one 
of the order parameters.

It is claimed in [18] that the relationships $\dot{\eta}_{i}=L_{i j} X_{j}$ that ensure that PTs between each pair of phases without the presence of the third phase for arbitrary $n$ can be achieved by a special choice of the matrix $L_{i j}$, which is a quite sophisticated nonlinear function of the order parameters. This matrix is ill-defined in the vicinity of each single phase and is substituted with other matrices. Here, we achieved a similar goal by using a simple penalizing term, which allows us to control (if it is observed in experiment $[29,31]$ ) and, if necessary, avoid appearance of the third phase. Also, as it is mentioned in [21], matrix $L_{i j}$ is "dangerous" from the point of view that it may make nonequilibrium microstructures, for which $X_{i} \neq 0$ is stationary.

In the very recent manuscript [21] conditions, which should satisfy the theory based on constraint $\sum \eta_{i}=1$, are explicitly formulated and satisfied, in particular, by improving the matrix $L_{i j}$ from [18]. Thus, currently theory [21] is the most consistent and justified one among theories with the constraint $\sum \eta_{i}=1$. While comparing it with our approach here (and to some extend in [32]), we have to state that our goal is broader, i.e., not just to exclude the third phase at the interface between two phases but to control its presence and degree of presence, including elimination of the third phase. Also, for phases with different properties, our expressions for matrices $L_{i j}$ and $\beta_{i j}$ should not be $\eta_{k}$-dependent, which makes all equations simpler. Also, our conditions 4, 5n, and 6n, related to instability conditions are not included in [21]. As we were informed in [62], our theory in [32] satisfies all relevant conditions formulated in [21]. Note that [21] contains much more detailed and critical analysis of the previous papers on the multiphase approach.

\section{References}

[1] V.I. Levitas, D.L. Preston, Three-dimensional Landau theory for multivariant stressinduced martensitic phase transformations. I. Austenite $\leftrightarrow$ Martensite, Phys. Rev. B. 66 (2002a) 134206.

[2] V.I. Levitas, D.L. Preston, Three-dimensional Landau theory for multivariant stressinduced martensitic phase transformations. II. Multivariant phase transformations and stress-space analysis, Phys. Rev. B 66 (2002b) 134207.

[3] A. Artemev, Y.M. Jin, A.G. Khachaturyan, Three-dimensional phase field model of proper martensitic transformation, Acta. Mat. 49 (2001) 1165-1177. 
[4] Y. M. Jin, A. Artemev, A.G. Khachaturyan, Three-dimensional phase field model of lowsymmetry martensitic transformation in polycrystal: Simulation of $\zeta_{2}$ martensite in $\mathrm{AuCd}$ alloys. Acta. Mat. 49 (2001a) 2309-2320.

[5] L.Q. Chen, Phase-field models for microstructure evolution. Annu. Rev. Mater. Res. 32 (2002) 113-140.

[6] Y. Wang, A. G. Khachaturyan, Multi-scale phase field approach to martensitic transformations. Mater. Sci. Eng. A. 438 (2006) 55-63.

[7] M. Mamivand, M.A. Zaeem, H. el Kadiri, A review on phase field modeling of martensitic phase transformation. Comp. Mater. Sci. 77 (2013) 304-311.

[8] V.I. Levitas, D.L. Preston, D.W. Lee, Three-dimensional Landau theory for multivariant stress-induced martensitic phase transformations. III. Alternative potentials, critical nuclei, kink solutions, and dislocation theory. Phys. Rev. B 68 (2003) 134201.

[9] I. Steinbach, F. Pezzolla, B. Nestler, M. Seelberg, R. Prieler, G.J. Schmitz, J.L.L. Rezende, A phase field concept for multiphase systems. Physica D 94 (1996) 13547.

[10] H. Garcke, B. Nestler,B. Stoth, On anisotropic order parameter models for multi-phase systems and their sharp interface limits, Physica D 115 (1998) 87.

[11] I. Steinbach, Phase-field models in materials science, Model. Simul. Mater. Sci. Eng. 17 (2009) 073001.

[12] R. Kobayashi, J. Warren, Modeling the formation and dynamics of polycrystals in 3D, Physica A 356 (2005) 127-132.

[13] G.I. Toth, J.R. Morris, L. Granasy, Ginzburg-Landau-Type Multiphase Field Model for Competing fcc and bcc Nucleation, Phys. Rev. Lett. 106 (2011) 045701.

[14] G.I. Toth, T. Pusztai, G. Tegze, L. Granasy, Amorphous Nucleation Precursor in Highly Nonequilibrium Fluids, Phys. Rev. Lett. 107 (2011) 175702.

[15] Y. Mishin, W.J. Boettinger, J.A. Warren, G.B. McFadden, Thermodynamics of grain boundary premelting in alloys. I. Phase-field modeling, Acta. Mater. 57 (2009) 3771.

[16] R. Folch, M. Plapp, Towards a quantitative phase-field model of two-phase solidification, Phys. Rev. E. 68(2003) 010602.

[17] R. Folch, M. Plapp, Quantitative phase-field modeling of two-phase growth, Phys. Rev. E. $72(2005) 011602$.

[18] P.C. Bollada, P.K. Jimack, A.M. Mullis, A New Approach to Multi-Phase Formulation for the Solidification of Alloys, Physica D 241 (2012) 816.

[19] V.I. Levitas, A.V. Idesman, D.L. Preston, Microscale simulation of evolution of martensitic microstructure, Phys. Rev. Let. 93 (2004) 105701.

[20] A.V. Idesman, V.I. Levitas, D.L. Preston, J-Y.Cho, Finite element simulations of martensitic phase transitions and microstructure based on strain softening model, J. Mech. Phys. Solids. 53 (2005) 495-523. 
[21] G.I. Toth, T. Pusztai, L. Granasy, Equilibrium and dynamics in multiphase-field theories: A comparative study and a consistent formulation, arXiv:1508.04311v1 [cond-mat.mtrlsci], 18 August 2015.

[22] A. Karma, W-J. Rappel, Phase-field method for computationally efficient modeling of solidification with arbitrary interface kinetics, Phys. Rev. E 53 (1996) R3017.

[23] A. Karma, W-J. Rappel, Quantitative phase-field modeling of dendritic growth in two and three dimensions, Phys. Rev. E 57 (1998) 4323.

[24] V.I. Levitas, K. Momeni, Solid-Solid Transformations via Nanoscale Intermediate Interfacial Phase: Multiple Structures, Scale, and Mechanics Effects, Acta. Mater. 65 (2014) 125.

[25] K. Momeni, V.I. Levitas, Propagating phase interface with intermediate interfacial phase: Phase field approach, Phys. Rev. B 89 (2014) 184102.

[26] K. Momeni, V.I. Levitas, J.A. Warren, The strong influence of internal stresses on the nucleation of a nanosized, deeply undercooled melt at a solid-solid interface, Nano Let. 15 (2015) 2298-2303.

[27] K. Momeni, V.I. Levitas, A Phase-Field Approach to Solid-Solid Phase Transformations via Intermediate Interfacial Phases under Stress Tensor, Int. J. Sol. Struc. 71 (2015) 39.

[28] V.I. Levitas, A.M. Roy, D.L. Preston, Multiple twinning and variant-variant transformations in martensite: Phase-field approach, Phys. Rev. B 88 (2013) 054113.

[29] V.I. Levitas, B.F. Henson, L.B. Smilowitz, B.W. Asay, Solid-solid phase transformation via virtual melt, significantly below the melting temperature, Phys. Rev. Lett. 92 (2004) 235702 .

[30] V.I. Levitas, B.F. Henson, L.B. Smilowitz, B.W. Asay, Solid-solid phase transformation via internal stress-induced virtual melting, significantly below the melting temperature. Application to HMX energetic crystal, J. Phys. Chem. B 110 (2006) 10105.

[31] V.I. Levitas, Z. Ren, Y. Zeng, Z. Zhang, G. Han, Crystal-crystal phase transformation via surface-induced virtual pre-melting, Phys. Rev. B 85 (2012) 220104.

[32] V.I. Levitas, A.M. Roy, Multiphase phase field theory for temperature- and stress-induced phase transformations. Phys. Rev. B 91 (2015) 174109.

[33] S-L. Wang, R.F. Sekerka, A.A. Wheeler, B.T. Murray, S.R. Coriell, R.J. Rraun, G.B. McFadden Thermodynamically-consistent phase-field models for solidification, Physica D 69 (1993) 189-200.

[34] V.I. Levitas, Phase field approach to martensitic phase transformations with large strains and interface stresses, J. Mech. Phys. Solids 70 (2014) 154.

[35] V.I. Levitas, Unambiguous Gibbs dividing surface for nonequilibrium finite-width interface: Static equivalence approach, Phys. Rev. B 89(2014) 094107.

[36] Ph. Boullay, D. Schryvers, R.V. Kohn, J.M. Ball, Lattice deformations at martensitemartensite interfaces in Ni-Al, Journal de Physique IV 11 (2001) 23. 
[37] M.A. Grinfeld, Thermodynamic Methods in the Theory of Heterogeneous Systems, Longman, Sussex (1991).

[38] P. Toledano, V. Dmitriev, Reconstructive Phase Transitions, World Scientific, New Jersey, (1996).

[39] J.C. Toledano, P. Toledano, The Landau Theory of Phase Transitions. World Scientific, (1988).

[40] E.K.H. Salje, Phase Transitions in Ferroelastic and Co-Elastic Crystals. Cambridge University Press, New York, (1990).

[41] A. Umantsev, Field Theoretic Method in Phase Transformations, Lecture Notes in Physics,Springer, New York 840 (2012).

[42] T. Ichitsubo, K. Tanaka, M. Koiwa, Yo. Yamazaki, Kinetics of cubic to tetragonal transformation under external field by thetime-dependent Ginzburg-Landau approach, Phys. Rev. B 62 (2000) 5435-5441.

[43] P.A. Lindgård, O.G. Mouritsen, Theory and Model for Martensitic Transformations, Phys. Rev. Let. 57 (1986) 2458-2461.

[44] A.A. Boulbitch, P. Toledano, Phase Nucleation of Elastic Defects in Crystals Undergoing a Phase Transition, Phy. Rev. Let. 81 (1998) 838.

[45] L.D. Landau, E.M. Lifshitz, Statistical Physics., Butterworth-Heinemann, Vol. 5 (3rd ed.), Oxford, (1980).

[46] A. Umantsev, Thermodynamic stability of phases and transition kinetics under adiabatic conditions, J. Chemical Phys. 96 (1992) 605-617.

[47] A. Umantsev, A.L. Roitburd, Nonisothermal relaxation in a nonlocal medium, Sov. Phys. Sol. St. 30 (1988) 651-655.

[48] Y.U. Wang, Y.M. Jin, A.M. Cuitio, A.G. Khachaturyan, Nanoscale phase field microelasticity theory of dislocations: model and 3D simulations, Acta. Mater. 49 (2001) 1847-1857.

[49] S.Y. Hu, L.Q. Chen, A phase-field model for evolving microstructures with strong elastic inhomogeneity, Acta. Mat. 49 (2001) 1879-1890.

[50] Y.U. Wang, J. Li, Phase field modeling of defects and deformation, Acta. Mater. 58 (2010) $1212-1235$.

[51] A. Hunter, I.J. Beyerlein, T.C. Germann, M. Koslowski, Influence of the stacking fault energy surface on partial dislocations in fcc metals with a three-dimensional phase field dislocations dynamics model, Phys. Rev. B 84 (2011) 144108.

[52] V.I. Levitas, V.A. Levin, K.M. Zingerman, E.I. Freiman, Displacive phase transitions at large strains: Phase-field theory and simulations, Phys. Rev. Lett. 103 (2009) 025702.

[53] V.I. Levitas, Phase-field theory for martensitic phase transformations at large strains, Int. J. Plast. 49 (2013) 85. 
[54] V.I. Levitas, M. Javanbakht, Advanced Phase-Field Approach to Dislocation Evolution, Phys. Rev. B 86 (2012) 140101.

[55] Y.S. Hwang, V.I. Levitas, Phase field simulation of kinetic superheating and melting of aluminum nanolayer irradiated by pico- and femtosecond laser, App. Phys. Let. 103 (2013) 263107.

[56] Y.S. Hwang, V.I. Levitas, Internal stress-induced melting below melting temperature at high-rate laser heating, App. Phys. Let. 104 (2014) 263106.

[57] V.I. Levitas, K. Samani, Coherent solid-liquid interface with stress relaxation in a phasefield approach to the melting/freezing transition, Phys. Rev. B 84 (2011) 140103.

[58] V.I. Levitas, K. Samani, Melting and solidification of nanoparticles: Scale effects, thermally activated surface nucleation, and bistable states, Phys. Rev. B 89 (2014) 075427.

[59] J.D. Clayton, J. Knap, A phase field model of deformation twinning: Nonlinear theory and numerical simulations, Physica D 240 (2011) 841.

[60] V.I. Levitas, M. Javanbakht, Surface tension and energy in multivariant martensitic transformations: Phase-field theory, simulations, and model of coherent interface, Phys. Rev. Let. 105 (2010) 165701.

[61] V.I. Levitas, M. Javanbakht, Phase-field approach to martensitic phase transformations: Effect of martensite martensite interface energy, Int. J. Mat. Res. 102 (2011) 652.

[62] G.I. Toth, private comun. (2015). 


\section{*Graphical Abstract}
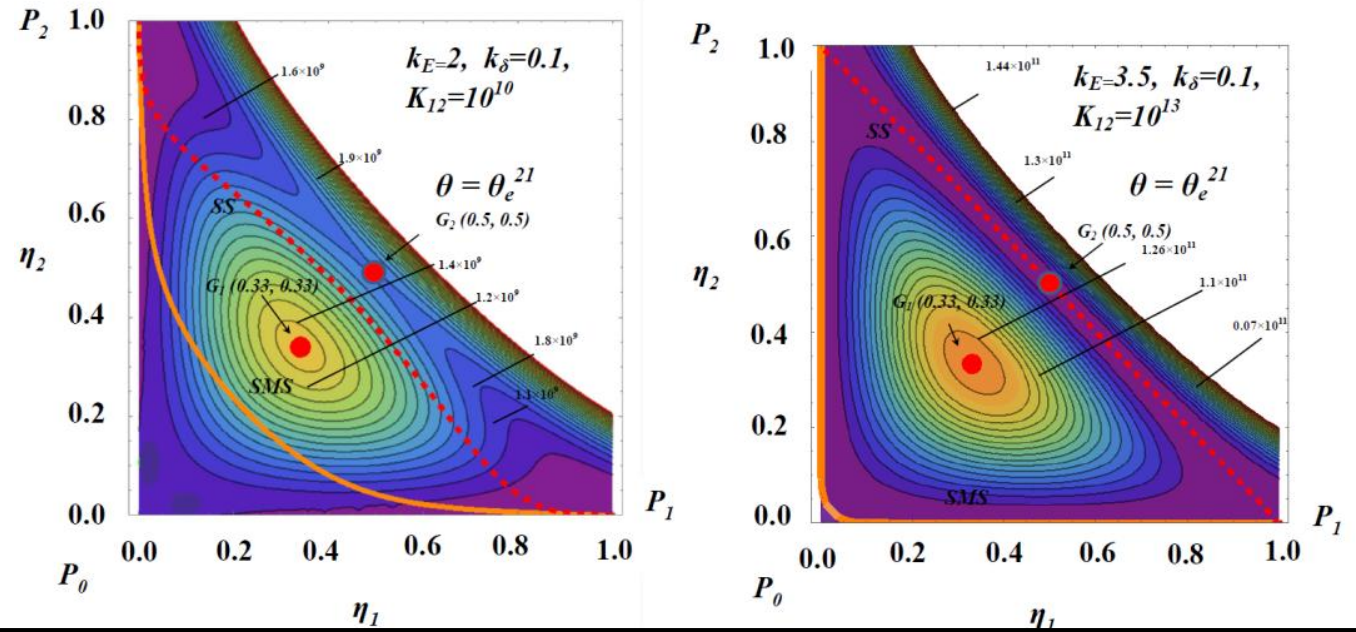